MATHEMATICS OF COMPUTATION

Volume 69, Number 231, Pages 965-986

S $0025-5718(00) 01186-8$

Article electronically published on March 3, 2000

\title{
A MIXED FORMULATION OF BOUSSINESQ EQUATIONS: ANALYSIS OF NONSINGULAR SOLUTIONS
}

\author{
M. FARHLOUL, S. NICAISE, AND L. PAQUET
}

\begin{abstract}
This paper is concerned with the mixed formulation of the Boussinesq equations in two-dimensional domains and its numerical approximation. The paper deals first with existence and uniqueness results, as well as the description of the regularity of any solution. The problem is then approximated by a mixed finite element method, where the gradient of the velocity and the gradient of the temperature, quantities of practical importance, are introduced as new unknowns. An existence result for the finite element solution and convergence results are proved near a nonsingular solution. Quasi-optimal error estimates are finally presented.
\end{abstract}

\section{INTRODUCTION}

Let $\Omega$ be a bounded domain of $\mathbb{R}^{2}$, with a Lipschitz continuous boundary $\Gamma$. We consider the stationary equations of thermohydraulics in the setting of Boussinesq approximation with Dirichlet boundary conditions for the velocity and mixed Dirichlet and Neumann boundary conditions for the temperature:

$$
\begin{cases}-\nu \Delta \mathbf{u}+(\mathbf{u} \cdot \nabla) \mathbf{u}+\alpha \theta+\nabla p=\mathbf{f} & \text { in } \Omega, \\ -k \Delta \theta+(\mathbf{u} \cdot \nabla) \theta=g & \text { in } \Omega, \\ \nabla \cdot \mathbf{u}=0 & \text { in } \Omega, \\ \mathbf{u}=0 & \text { on } \Gamma, \\ \theta=0 & \text { on } \Gamma_{D}, \\ \frac{\partial \theta}{\partial \mathbf{n}}=0 & \text { on } \Gamma_{N},\end{cases}
$$

where $\mathbf{u}$ is the velocity field, $p$ the pressure, $\theta$ the temperature, and

$$
(\mathbf{u} \cdot \nabla) \mathbf{u}=\left(\sum_{j=1}^{2} u_{j} \frac{\partial u_{1}}{\partial x_{j}}, \sum_{j=1}^{2} u_{j} \frac{\partial u_{2}}{\partial x_{j}}\right), \quad \nabla \cdot \mathbf{u}=\frac{\partial u_{1}}{\partial x_{1}}+\frac{\partial u_{2}}{\partial x_{2}} .
$$

$\Gamma_{D}$ is a nonempty open part of $\Gamma, \Gamma_{N}=\Gamma \backslash \bar{\Gamma}_{D}$, $\mathbf{n}$ denotes the unit outward normal to $\Gamma$, and by $\frac{\partial}{\partial \mathbf{n}}$, we mean the exterior normal derivative. We suppose that the right-hand sides of (1) are square-integrable in $\Omega$, i.e., we impose that $\mathbf{f} \in\left(L^{2}(\Omega)\right)^{2}$ and $g \in L^{2}(\Omega)$. The coefficients $\nu$ and $k$ in (1) are assumed to be positive; $\nu$ is

Received by the editor May 9, 1997 and, in revised form, July 15, 1998.

1991 Mathematics Subject Classification. Primary 65N30. 
called the kinematic viscosity and $k$ the thermal diffusivity. $\alpha$ denotes a constant vector in $\mathbb{R}^{2}$, the term $\alpha \theta$ in the first equation is related to the buoyancy forces; in our context $\alpha$ is arbitrary while usually in physical contexts it is parallel to the vertical axis.

Recently, Paquet [14 and Bernardi et al. [1] have studied systems of equations similar to (1). They have proved that this problem has at least one solution. In [1], the authors also show that under some very restrictive hypothesis on the data, this solution is unique; moreover they analyze the corresponding discrete problem by classical finite element methods.

Let us mention that the first equation of (1) is slightly different from the one considered in Bernardi et al. [1. However, in Section 2 we adapt the proof of the existence results obtained by Bernardi et al. to our system (1). We further give sufficient conditions on the data $\mathbf{f}$ and $g$ insuring uniqueness. The difference between system (11) and the system considered in 14 comes from the boundary conditions since there thermocapillarity effects are taken into account. Note also that in [14] existence results are based on fixed point arguments (as in [11]), while here we use the degree theory as in 1 .

In Section 3, we analyze the regularities of the solutions $\mathbf{u}, p$ and $\theta$. Namely assuming that $\Omega$ has a polygonal boundary, we give the optimal regularities of the solutions (in the spirit of [13, 4, 5]). As a consequence, we deduce sufficient geometrical conditions insuring the regularity $H^{2}(\Omega)$ for $\mathbf{u}, \theta$ and $H^{1}(\Omega)$ for $p$. As usual such results are useful for finite element analysis. Here they are also used to check the equivalence between the classical weak formulation of problem (1) and its mixed formulation.

In Section 4 we consider a mixed formulation of problem (1), where the gradient of the velocity and the gradient of the temperature are introduced as new unknowns. Thus the problem (11) can be formulated as

$$
\begin{cases}\boldsymbol{\sigma}=\nu \nabla \mathbf{u} & \text { in } \Omega, \\ \nabla \cdot(\boldsymbol{\sigma}-p \boldsymbol{\delta})-\frac{1}{\nu} \boldsymbol{\sigma} \cdot \mathbf{u}-\alpha \theta+\mathbf{f}=0 & \text { in } \Omega, \\ \nabla \cdot \mathbf{u}=0 & \text { in } \Omega, \\ \xi=k \nabla \theta & \text { in } \Omega, \\ \nabla \cdot \xi-\frac{1}{k} \mathbf{u} \cdot \xi+g=0 & \text { in } \Omega, \\ \mathbf{u}=0 & \text { on } \Gamma, \\ \theta=0 & \text { on } \Gamma_{D}, \\ \frac{\partial \theta}{\partial \mathbf{n}}=0 & \text { on } \Gamma_{N},\end{cases}
$$

where $\boldsymbol{\delta}$ is the identity tensor,

$$
\nabla \mathbf{u}=\left(\frac{\partial u_{i}}{\partial x_{j}}\right)_{1 \leq i, j \leq 2}, \quad \boldsymbol{\sigma} \cdot \mathbf{u}=\left(\sum_{j=1}^{2} \sigma_{1 j} u_{j}, \sum_{j=1}^{2} \sigma_{2 j} u_{j}\right)
$$


and for a tensor $\boldsymbol{\tau}$,

$$
\nabla \cdot \boldsymbol{\tau}=\left(\frac{\partial \tau_{11}}{\partial x_{1}}+\frac{\partial \tau_{12}}{\partial x_{2}}, \frac{\partial \tau_{21}}{\partial x_{1}}+\frac{\partial \tau_{22}}{\partial x_{2}}\right) .
$$

Clearly, a classical finite element method may be used for the approximation of (11) as used in 1. However in many applications, the knowledge of the gradient of the velocity and the gradient of the temperature $(\boldsymbol{\sigma}$ and $\xi)$ is of particular importance. In such cases, the use of a mixed finite element method might be preferred as long as it provides a better accuracy for $\boldsymbol{\sigma}$ and $\xi$.

The mixed finite element that we will consider in this paper, for problem (2), is a combination of the one that we have analyzed in [9] for the Navier-Stokes problem and the lowest degree Raviart-Thomas finite element [15] for Dirichlet's problem. Assuming that $(\mathbf{u}, p, \theta)$ is an isolated solution of (1) and that the mesh width $h$ is small enough, we will prove in Section 5 that the discretized scheme has a solution and we will find optimal bounds for the error of the same order on $\mathbf{u}, p, \theta, \boldsymbol{\sigma}$ and $\xi$. Let us mention that a numerical test confirming the theoretical estimates has been performed in [10].

We close this introduction by pointing out that the analysis of the mixed finite element for problem (2) with nonhomogeneous boundary conditions presents more technical difficulties. This problem is left for the future.

\section{EXIstence OF A SOLUTiON \\ TO THE STEADY-STATE BOUSSINESQ EQUATIONS \\ AND A UNIQUENESS RESULT}

We first introduce some notation that will be used in the following. $H^{s}(\Omega)$, $\stackrel{\circ}{H}^{s}(\Omega), s \in \mathbb{R}$, denote the standard Sobolev spaces normed by $\|\cdot\|_{s, \Omega}[13$. In particular $H^{\frac{1}{2}}(\Gamma)$ is the space of traces of functions in $H^{1}(\Omega)$ and $H^{-\frac{1}{2}}(\Gamma)$ is its dual space. The inner product of $L^{2}(\Omega):=H^{0}(\Omega)$ is denoted by $(.,$.$) . Since no$ confusion can arise, we use the same notation for the corresponding norms and inner products on $\mathbf{L}^{s}(\Omega)=L^{s}(\Omega) \times L^{s}(\Omega), \mathbf{H}^{s}(\Omega)=H^{s}(\Omega) \times H^{s}(\Omega)$, etc. $L_{0}^{2}(\Omega)$ stands for the subspace of $L^{2}(\Omega)$ consisting of functions with zero mean value over $\Omega$. We will frequently use the spaces

$$
H(\operatorname{div}, \Omega)=\left\{\mathbf{v} \in \mathbf{L}^{2}(\Omega): \nabla \cdot \mathbf{v} \in L^{2}(\Omega)\right\}
$$

and

$$
\mathbf{H}(\operatorname{div}, \Omega)=H(\operatorname{div}, \Omega) \times H(\operatorname{div}, \Omega),
$$

which are equipped respectively with the norms

$$
\|\mathbf{v}\|_{H(\operatorname{div}, \Omega)}=\left\{\|\mathbf{v}\|_{0, \Omega}^{2}+\|\nabla \cdot \mathbf{v}\|_{0, \Omega}^{2}\right\}^{\frac{1}{2}}
$$

and

$$
\|\boldsymbol{\tau}\|_{\mathbf{H}(\operatorname{div}, \Omega)}=\left\{\|\boldsymbol{\tau}\|_{0, \Omega}^{2}+\|\nabla \cdot \boldsymbol{\tau}\|_{0, \Omega}^{2}\right\}^{\frac{1}{2}} .
$$

Note that the trace operator $\mathbf{v} \longrightarrow \mathbf{v} \cdot \mathbf{n}$ is a continuous mapping of $H(\operatorname{div}, \Omega)$ onto $H^{-\frac{1}{2}}(\Gamma)$ where $\mathbf{n}$ denotes the unit outward normal to $\Gamma$ (cf. [12]).

The purpose of this section is to prove the existence of a solution to the Boussinesq equations (11). We will also give sufficient conditions insuring the uniqueness of the solution. In particular, it will be shown that the solution is unique if the right-hand sides are sufficiently small. 
Let us first start with the existence problem. The arguments involved consist in a slight variant of those in [1], the difference coming from the fact that in [1] it is assumed that $\mathbf{f}=0$ in the first equation of (1).

Let us now derive the variational formulation of our problem. We begin by introducing the following functional spaces:

$$
\begin{aligned}
\mathcal{V} & =\left\{\mathbf{v} \in\left(\stackrel{\circ}{H}^{1}(\Omega)\right)^{2} ; \operatorname{div} \mathbf{v}=0 \text { in } \Omega\right\} \\
H_{\star}^{1}(\Omega) & =\left\{\eta \in H^{1}(\Omega) ;\left.\eta\right|_{\Gamma_{D}}=0\right\}
\end{aligned}
$$

endowed with the norms

$$
\begin{aligned}
\|\mathbf{v}\|^{2} & =\int_{\Omega}\left(\left|\operatorname{grad} v_{1}\right|^{2}+\left|\operatorname{grad} v_{2}\right|^{2}\right) d x \quad \forall \mathbf{v}=\left(v_{1}, v_{2}\right) \in \mathcal{V}, \\
\|\eta\|^{2} & =\int_{\Omega}|\operatorname{grad} \eta|^{2} d x \quad \forall \eta \in H_{\star}^{1}(\Omega) .
\end{aligned}
$$

Performing a formal integration by part in the two first equations of (1) against some $\mathbf{v} \in \mathcal{V}$ and $\eta \in H_{\star}^{1}(\Omega)$, respectively, we get the following weak formulation of problem (11). Find a pair $U=(\mathbf{u}, \theta) \in X:=\mathcal{V} \times H_{\star}^{1}(\Omega)$ such that for every $V=(\mathbf{v}, \eta) \in X:$

$$
\left\{\begin{array}{l}
\nu \int_{\Omega} \operatorname{grad} \mathbf{u} \cdot \operatorname{grad} \mathbf{v} d x+\int_{\Omega}(\mathbf{u} \nabla) \mathbf{u} \cdot \mathbf{v} d x+\int_{\Omega} \alpha \theta \cdot \mathbf{v} d x=\int_{\Omega} \mathbf{f} \cdot \mathbf{v} d x \\
k \int_{\Omega} \operatorname{grad} \theta \cdot \operatorname{grad} \eta d x+\int_{\Omega}(\mathbf{u} \nabla) \theta \eta d x=\int_{\Omega} g \eta d x
\end{array}\right.
$$

We first need some a priori estimates whose proof is similar to the one of Proposition 2.1 of [1].

Proposition 2.1. Let $U=(\mathbf{u}, \theta) \in X$ be a solution of (5). Then we have

$$
\begin{aligned}
\|\theta\| & \leq \frac{\gamma}{k} \\
\|\mathbf{u}\| & \leq|\alpha| \frac{\mathcal{P} \mathcal{P}^{\star}}{\nu}\|\theta\|+\frac{\mathcal{P}}{\nu}\|\mathbf{f}\|_{0, \Omega},
\end{aligned}
$$

where

$$
\begin{aligned}
& \gamma=\sup _{\eta \in H_{\star}^{1}(\Omega) \backslash\{0\}} \frac{\int_{\Omega} g \eta d x}{\|\eta\|}, \\
& \mathcal{P}=\sup _{\substack{\stackrel{\circ}{1}^{1}(\Omega) \backslash\{0\}\\
}} \frac{\|\eta\|_{0, \Omega}}{\|\eta\|}, \quad \mathcal{P}^{\star}=\sup _{\eta \in H_{\star}^{1}(\Omega) \backslash\{0\}} \frac{\|\eta\|_{0, \Omega}}{\|\eta\|} .
\end{aligned}
$$

Consequently, we also have

$$
\|\mathbf{u}\| \leq|\alpha| \frac{\mathcal{P} \mathcal{P}^{\star} \gamma}{\nu k}+\frac{\mathcal{P}}{\nu}\|\mathbf{f}\|_{0, \Omega}
$$

In particular, the solutions $U=(\mathbf{u}, \theta) \in X$ of (5) are a priori bounded.

As in [1], we are going to use degree theory. Let us then endow $X$ with the following inner product. For every $U=(\mathbf{u}, \theta), V=(\mathbf{v}, \eta) \in X$, we take

$$
(U, V):=\int_{\Omega} \operatorname{grad} \mathbf{u} \cdot \operatorname{grad} \mathbf{v} d x+\int_{\Omega} \operatorname{grad} \theta \cdot \operatorname{grad} \eta d x
$$


For every $\alpha \in \mathbb{R}^{2}$ and $\mathbf{f} \in \mathbf{L}^{2}(\Omega)$, we define a nonlinear map $\Phi^{\alpha, \mathbf{f}}$ from $X$ into $X$ as follows. For every $U=(\mathbf{u}, \theta), V=(\mathbf{v}, \eta) \in X$ :

$$
\begin{aligned}
\left(\Phi^{\alpha, \mathbf{f}} U, V\right) & =\int_{\Omega} \operatorname{grad} \mathbf{u} \cdot \operatorname{grad} \mathbf{v} d x+\frac{1}{\nu} \int_{\Omega}(\mathbf{u} \nabla) \mathbf{u} \cdot \mathbf{v} d x \\
& +\frac{1}{\nu} \int_{\Omega} \alpha \theta \cdot \mathbf{v} d x-\frac{1}{\nu} \int_{\Omega} \mathbf{f} \cdot \mathbf{v} d x \\
& +\int_{\Omega} \operatorname{grad} \theta \cdot \operatorname{grad} \eta d x+\frac{1}{k} \int_{\Omega}(\mathbf{u} \nabla) \theta \eta d x-\frac{1}{k} \int_{\Omega} g \eta d x .
\end{aligned}
$$

In other words, the right-hand side of (11) defines a continuous linear form on $X$, which by the Riesz representation theorem is the inner product with one and only one element of $X$, called $\Phi^{\alpha, \mathbf{f}} U$. Clearly, $U=(\mathbf{u}, \theta) \in X$ is a solution of (15) if and only if $\Phi^{\alpha, \mathbf{f}} U=0$.

We can also define another nonlinear map $\mathcal{F}^{\alpha, \mathbf{f}}$ from $X$ into itself by

$$
\left(\mathcal{F}^{\alpha, \mathbf{f}} U, V\right)=\left(\Phi^{\alpha, \mathbf{f}} U, V\right)-(U, V) \quad \forall U, V \in X .
$$

It results from (11) and (12) that $\Phi^{\alpha, \mathbf{f}}=I+\mathcal{F}^{\alpha, \mathbf{f}}$; consequently the equation $\Phi^{\alpha, \mathbf{f}} U=0$ is equivalent to $-\mathcal{F}^{\alpha, \mathbf{f}} U=U$. Therefore, we are reduced to find a fixed point for the mapping $-\mathcal{F}^{\alpha, \mathbf{f}}$.

In the following, $\mathcal{O}$ will denote a fixed bounded open set of $X$ containing the set

$$
\left\{U=(\mathbf{u}, \theta) \in X ;\|\theta\| \leq \frac{\gamma}{k},\|\mathbf{u}\| \leq|\alpha| \frac{\mathcal{P} \mathcal{P}^{\star} \gamma}{\nu k}+\frac{\mathcal{P}}{\nu}\|\mathbf{f}\|_{0, \Omega}\right\}
$$

Accordingly, by Proposition 2.1, we are sure that

$$
\forall U \in \partial \mathcal{O}: \Phi^{\alpha, \mathbf{f}} U \neq 0 .
$$

To be allowed to speak about the degree of $\Phi^{\alpha, \mathbf{f}}$ with respect to $\mathcal{O}$ and 0 , we must show that $\mathcal{F}^{\alpha, \mathbf{f}}: \overline{\mathcal{O}} \rightarrow X$ is completely continuous [16, p. 184]. As the Riesz isomorphism from $X^{\prime}$ into $X$ is continuous, it suffices to show that the mapping $\overline{\mathcal{O}} \rightarrow X^{\prime}: U \rightarrow\left(\mathcal{F}^{\alpha, \mathbf{f}} U, \cdot\right)$ is completely continuous. Now from 12$),\left(\mathcal{F}^{\alpha, \mathbf{f}} U, \cdot\right)$ extends naturally to a continuous linear form on $\stackrel{\circ}{\mathbf{H}}^{1}(\Omega) \times H_{\star}^{1}(\Omega)$. Denoting this extension by $\left(\mathcal{F}^{\alpha, \mathbf{f}} U, \cdot\right)^{\tilde{2}}$, it is clear that it suffices to show that the mapping: $\overline{\mathcal{O}} \rightarrow$ $\left(\stackrel{\circ}{\mathbf{H}}^{1}(\Omega) \times H_{\star}^{1}(\Omega)\right)^{\prime}=H^{-1}(\Omega) \times H_{\star}^{1}(\Omega)^{\prime}: U \rightarrow\left(\mathcal{F}^{\alpha, \mathbf{f}} U, \cdot\right)^{\tilde{2}}$ is completely continuous. This is easily shown by using the same arguments as in the proof of Proposition 2.5 of 11 based on the compact imbedding of $H^{1}(\Omega)$ into $L^{4}(\Omega)$. This fact and (14) show that the (Leray-Schauder) degree of $\Phi^{\alpha, \mathbf{f}}$ with respect to $\mathcal{O}$ and 0 is well defined. In conformity with [16, p. 184], we denote it by $d\left[\Phi^{\alpha, f}, \mathcal{O}, 0\right]$. By the existence theorem of Kronecker [16, pp. 176, 184], to prove that there exists $U \in \mathcal{O}$ solution of $\Phi^{\alpha, \mathbf{f}} U=0$, it suffices to show that $d\left[\Phi^{\alpha, \mathbf{f}}, \mathcal{O}, 0\right] \neq 0$.

Proposition 2.2. If the bounded open set $\mathcal{O}$ is taken sufficiently large, then $d\left[\Phi^{0,0}, \mathcal{O}, 0\right] \in\{1,-1\}$.

Proof. The ideas of the proof follow those of Proposition 2.5 of [1] because here $\mathbf{f}=0$ with even the simplification that we only consider homogeneous Neumann boundary conditions.

Theorem 2.3. Under the assumptions of Proposition 2.2, we also have $d\left[\Phi^{\alpha, \mathbf{f}}, \mathcal{O}, 0\right] \in\{1,-1\}$ and consequently problem (5) has at least one solution. 
Proof. We modify the homotopy introduced in Theorem 2.7 of [1] in order to take into account the nonzero datum $\mathbf{f}$. Here we take

$$
\psi: \overline{\mathcal{O}} \times[0,1] \rightarrow X:(U, t) \rightarrow \Phi^{t \alpha, t \mathbf{f}} U .
$$

By (13) and Proposition $\left[2.1\right.$ we have $\Phi^{t \alpha, t \mathbf{f}} U \neq 0$, for every $t \in[0,1]$ and every $U \in \partial \mathcal{O}$.

Clearly $\psi(U, t)=U+\mathcal{F}^{t \alpha, t \mathbf{f}} U$. Let us then define the mapping

$$
F: \overline{\mathcal{O}} \times[0,1] \rightarrow X:(U, t) \rightarrow \mathcal{F}^{t \alpha, t \mathbf{f}} U .
$$

If we show that $F$ is completely continuous, then it will follow from the invariance of the degree by such homotopy [16, p. 185] and from Proposition [2.2 that

$$
d\left[\Phi^{\alpha, \mathbf{f}}, \mathcal{O}, 0\right]=d\left[\Phi^{0,0}, \mathcal{O}, 0\right] \in\{1,-1\}
$$

and the theorem will be proved.

By the continuity of the Riesz isomorphism, it suffices to show that the mapping $\overline{\mathcal{O}} \times[0,1] \rightarrow X^{\prime}:(U, t) \rightarrow\left(\mathcal{F}^{t \alpha, t \mathbf{f}} U, \cdot\right)$ is completely continuous. As before denote by $\tilde{F}$ the natural extension of $F$ to $\mathbf{H}^{-1}(\Omega) \times H_{\star}^{1}(\Omega)^{\prime}$ defined by

$$
\tilde{F}: \overline{\mathcal{O}} \times[0,1] \rightarrow \mathbf{H}^{-1}(\Omega) \times H_{\star}^{1}(\Omega)^{\prime}:(U, t) \rightarrow\left(\mathcal{F}^{t \alpha, t \mathbf{f}} U, \cdot \tilde{)} .\right.
$$

The restriction mapping from $\stackrel{\circ}{\mathbf{H}}^{1}(\Omega) \times H_{\star}^{1}(\Omega)$ to $X$ being continuous, it suffices to prove that $\tilde{F}$ is completely continous. Now from (12), we have

$$
\begin{aligned}
\left(\mathcal{F}^{t \alpha, t \mathbf{f}}(\mathbf{u}, \theta),(\mathbf{v}, \eta)\right)^{\tilde{2}=} & \frac{1}{\nu} \int_{\Omega}(\mathbf{u} \nabla) \mathbf{u} \cdot \mathbf{v} d x+\frac{1}{k} \int_{\Omega}(\mathbf{u} \nabla) \theta \eta d x \\
& -\frac{1}{k} \int_{\Omega} g \eta d x+t\left[\frac{1}{\nu} \int_{\Omega} \alpha \theta \cdot \mathbf{v} d x-\frac{1}{\nu} \int_{\Omega} \mathbf{f} \cdot \mathbf{v} d x\right] .
\end{aligned}
$$

We already know that the first three terms of this right-hand side define a completely continuous operator from $\overline{\mathcal{O}} \times[0,1]$ to $\mathbf{H}^{-1}(\Omega) \times H_{\star}^{1}(\Omega)^{\prime}$. The last term is $t$ times a constant term so that it trivially defines a completely continuous operator (as a consequence of the compactness of $[0,1]$ in $\mathbb{R}$ ). The last but one term $t \frac{1}{\nu} \int_{\Omega} \alpha \theta \cdot \mathbf{v} d x$ defines also a completely continuous operator due to the compact imbedding of $H_{\star}^{1}(\Omega)$ into $L^{2}(\Omega)$. This shows that $\tilde{F}$ is completely continuous, and thus $F$ too.

Now we are going to state and prove a sufficient condition for the uniqueness. This condition involved the data $\mathbf{f}, g$ as well as the physical constants $\nu, k$ and $\alpha$. Let us first prove some technical estimates.

Lemma 2.4. Let $U_{1}=\left(\mathbf{u}_{1}, \theta_{1}\right), U_{2}=\left(\mathbf{u}_{2}, \theta_{2}\right) \in X$ be two solutions of (5). Then they satisfy

$$
\begin{aligned}
\nu\left\|\mathbf{u}_{1}-\mathbf{u}_{2}\right\|^{2}= & -\int_{\Omega}\left[\left(\left(\mathbf{u}_{1}-\mathbf{u}_{2}\right) \cdot \nabla\right) \mathbf{u}_{2}\right] \cdot\left(\mathbf{u}_{1}-\mathbf{u}_{2}\right) d x \\
& -\int_{\Omega} \alpha\left(\theta_{1}-\theta_{2}\right) \cdot\left(\mathbf{u}_{1}-\mathbf{u}_{2}\right) d x \\
k\left\|\theta_{1}-\theta_{2}\right\|^{2} & =-\int_{\Omega}\left[\left(\mathbf{u}_{1}-\mathbf{u}_{2}\right) \cdot \nabla\right] \theta_{2}\left(\theta_{1}-\theta_{2}\right) d x .
\end{aligned}
$$


Proof. Applying successively the first identity of (5) with $\mathbf{u}=\mathbf{u}_{1}, \theta=\theta_{1}, \mathbf{v}=$ $\mathbf{u}_{1}-\mathbf{u}_{2}$ and $\mathbf{u}=\mathbf{u}_{2}, \theta=\theta_{2}, \mathbf{v}=\mathbf{u}_{1}-\mathbf{u}_{2}$ and subtracting the two obtained identities, we get

$$
\begin{aligned}
\nu\left\|\mathbf{u}_{1}-\mathbf{u}_{2}\right\|^{2} & +\int_{\Omega}\left[\left(\mathbf{u}_{1} \nabla\right) \mathbf{u}_{1}-\left(\mathbf{u}_{2} \nabla\right) \mathbf{u}_{2}\right] \cdot\left(\mathbf{u}_{1}-\mathbf{u}_{2}\right) d x \\
& +\int_{\Omega} \alpha\left(\theta_{1}-\theta_{2}\right) \cdot\left(\mathbf{u}_{1}-\mathbf{u}_{2}\right) d x=0 .
\end{aligned}
$$

By Green's theorem and the condition $\operatorname{div} \mathbf{u}_{1}=0$ in $\Omega$, it follows that

$$
\int_{\Omega}\left(\mathbf{u}_{1} \nabla\right)\left(\mathbf{u}_{1}-\mathbf{u}_{2}\right) \cdot\left(\mathbf{u}_{1}-\mathbf{u}_{2}\right) d x=0 .
$$

Using this identity into (17), we obtain (15).

The identity (16) is proved similarly using the second identity of (5)).

Lemma 2.5. Under the assumptions of Lemma 2.4, we have

$$
\left\|\theta_{1}-\theta_{2}\right\| \leq \frac{\mathcal{S S}^{\star}}{k^{2}}\|g\|_{H_{\star}^{1}(\Omega)^{\prime}}\left\|\mathbf{u}_{1}-\mathbf{u}_{2}\right\|,
$$

where $\mathcal{S}$ and $\mathcal{S}^{\star}$ denote the Sobolev constants

$$
\begin{aligned}
& \mathcal{S}=\sup _{\substack{\mathcal{O}^{1} \\
H^{1}(\Omega) \backslash\{0\}}} \frac{\|\eta\|_{0,4, \Omega}}{\|\eta\|}, \\
& \mathcal{S}^{\star}=\sup _{\eta \in H_{\star}^{1}(\Omega) \backslash\{0\}} \frac{\|\eta\|_{0,4, \Omega}}{\|\eta\|} .
\end{aligned}
$$

Proof. Applying successively Hölder's inequality, the definition of $\mathcal{S}$ and $\mathcal{S}^{\star}$ and Proposition 2.1, we obtain

$$
\begin{aligned}
\mid \int_{\Omega} & {\left[\left(\mathbf{u}_{1}-\mathbf{u}_{2}\right) \cdot \nabla\right] \theta_{2}\left(\theta_{1}-\theta_{2}\right) d x \mid } \\
& \leq \sum_{j=1,2}\left\|\left(\mathbf{u}_{1}-\mathbf{u}_{2}\right)_{j}\right\|_{0,4, \Omega}\left\|\frac{\partial \theta_{2}}{\partial x_{j}}\right\|_{0, \Omega}\left\|\theta_{1}-\theta_{2}\right\|_{0,4, \Omega} . \\
& \leq \mathcal{S} \mathcal{S}^{\star}\left\|\mathbf{u}_{1}-\mathbf{u}_{2}\right\|\left\|\theta_{2}\right\|\left\|\theta_{1}-\theta_{2}\right\| \\
& \leq \frac{\mathcal{S} \mathcal{S}^{\star}}{k}\|g\|_{H_{\star}^{1}(\Omega)^{\prime}}\left\|\mathbf{u}_{1}-\mathbf{u}_{2}\right\|\left\|\theta_{1}-\theta_{2}\right\|
\end{aligned}
$$

The result follows using this last estimate in (16).

Corollary 2.6. Under the assumptions of Lemma 2.4, we have

$$
\left|\int_{\Omega} \alpha\left(\theta_{1}-\theta_{2}\right) \cdot\left(\mathbf{u}_{1}-\mathbf{u}_{2}\right) d x\right| \leq \frac{|\alpha| \mathcal{P} \mathcal{P}^{\star} \mathcal{S} \mathcal{S}^{\star}}{k^{2}}\|g\|_{H_{\star}^{1}(\Omega)^{\prime}}\left\|\mathbf{u}_{1}-\mathbf{u}_{2}\right\|^{2} .
$$

Proof. This follows from the Cauchy-Schwarz inequality, (9), and the estimate of Lemma 2.5

Lemma 2.7. Under the assumptions of Lemma 2.4, we have

$$
\begin{aligned}
& \left|\int_{\Omega}\left[\left(\left(\mathbf{u}_{1}-\mathbf{u}_{2}\right) \cdot \nabla\right) \mathbf{u}_{2}\right] \cdot\left(\mathbf{u}_{1}-\mathbf{u}_{2}\right) d x\right| \\
& \quad \leq \frac{\mathcal{S}^{2}}{\nu}\left\|\mathbf{u}_{1}-\mathbf{u}_{2}\right\|^{2}\left[|\alpha| \frac{\mathcal{P} \mathcal{P}^{\star}}{k}\|g\|_{H_{\star}^{1}(\Omega)^{\prime}}+\|\mathbf{f}\|_{\mathcal{V}^{\prime}}\right] .
\end{aligned}
$$


Proof. As (7), one can show that

$$
\left\|\mathbf{u}_{2}\right\| \leq|\alpha| \frac{\mathcal{P} \mathcal{P}^{\star}}{\nu}\left\|\theta_{2}\right\|+\frac{1}{\nu}\|\mathbf{f}\|_{\mathcal{V}^{\prime}}
$$

The desired estimate follows from the Cauchy-Schwarz inequality, the above inequality, the definition of $\mathcal{S}$, and the estimate (6) with (8)).

We are now ready to prove our uniqueness result (compare with the condition (2.25) of [1]).

Theorem 2.8. If $\kappa:=\frac{\mathcal{S}^{2}}{\nu^{2}}\|\mathbf{f}\|_{\mathcal{V}^{\prime}}+|\alpha| \mathcal{S} P \mathcal{P}^{\star}\left[\frac{\mathcal{S}}{\nu^{2} k}+\frac{\mathcal{S}^{\star}}{k^{2} \nu}\right]\|g\|_{H_{\star}^{1}(\Omega)^{\prime}}<1$, then problem (5) has a unique solution.

Proof. Let $\left(\mathbf{u}_{1}, \theta_{1}\right)$ and $\left(\mathbf{u}_{2}, \theta_{2}\right)$ be two solutions of problem (5). Then by (15), Corollary 2.6 and Lemma 2.7, one gets

$$
\left\|\mathbf{u}_{1}-\mathbf{u}_{2}\right\|^{2} \leq \kappa\left\|\mathbf{u}_{1}-\mathbf{u}_{2}\right\|^{2}
$$

If $\mathbf{u}_{1} \neq \mathbf{u}_{2}$, we may divide the two sides of this inequality by $\left\|\mathbf{u}_{1}-\mathbf{u}_{2}\right\|^{2}$, which contradicts the hypothesis. Consequently, $\mathbf{u}_{1}=\mathbf{u}_{2}$ and by Lemma 2.5, we deduce that $\theta_{1}=\theta_{2}$.

Corollary 2.9. If $\kappa_{1}:=\frac{\mathcal{S}^{2} \mathcal{P}}{\nu^{2}}\|\mathbf{f}\|_{0, \Omega}+|\alpha| \mathcal{S} P \mathcal{P}^{\star 2}\left[\frac{\mathcal{S}}{\nu^{2} k}+\frac{\mathcal{S}^{\star}}{k^{2} \nu}\right]\|g\|_{0, \Omega}<1$, then problem (5) has a unique solution.

Proof. By the Cauchy-Schwarz inequality and (9), we have

$$
\|\mathbf{f}\|_{\mathcal{V}^{\prime}} \leq \mathcal{P}\|\mathbf{f}\|_{0, \Omega}, \quad\|g\|_{H_{\star}^{1}(\Omega)^{\prime}} \leq \mathcal{P}^{\star}\|g\|_{0, \Omega}
$$

Consequently, with the notation from Theorem [2.8, one has $\kappa \leq \kappa_{1}$ and the result follows from Theorem 2.8 and the assumption $\kappa_{1}<1$.

\section{Regularity of the solutions}

From now on, we suppose that $\Omega$ is a plane domain with polygonal boundary. More precisely, it is assumed that $\Omega$ is a simply connected domain and that its boundary $\Gamma$ is the union of a finite number of linear segments $\bar{\Gamma}_{j}, 1 \leq j \leq n_{e}$ (it is more convenient to assume that $\Gamma_{j}$ is an open linear segment [13, p. 182]). We further fix a partition of $\left\{1, \ldots, n_{e}\right\}$ into two subsets $\mathcal{N}$ and $\mathcal{D}$. The union of the $\Gamma_{j}$ with $j \in \mathcal{D}$ is denoted by $\Gamma_{D}$ and similarly the union of the $\Gamma_{j}$ with $j \in \mathcal{N}$ is denoted by $\Gamma_{N}$. As before, we assume that $\Gamma_{D}$ is not empty.

The aim of this section is to describe the regularity of any solution $(\mathbf{u}, p, \theta) \in$ $\mathcal{V} \times L_{0}^{2}(\Omega) \times H_{\star}^{1}(\Omega)$ of problem (5). We shall see that this regularity is related to the singularities of the solution of the Stokes problem with Dirichlet boundary condition in $\Omega$ and the solution of the Laplace equation with mixed boundary conditions. To recall the regularity results about these problems obtained in [13, 4, 5], let us introduce the following notation. Let $S_{j}, j=1, \ldots, n_{e}$, denote the set of vertices of $\Omega$ and let $\omega_{j}$ denote the interior opening of $\Omega$ at $S_{j}$. Then the singular exponents of the Stokes problem near $S_{j}$ are the roots $\lambda \in \mathbf{C} \backslash\{0\}$ of

$$
\sin ^{2}\left(\lambda \omega_{j}\right)-\lambda^{2} \sin ^{2} \omega_{j}=0
$$

(see [5] for more details). Let us set $\xi_{S}\left(\omega_{j}\right)=\min \{\Re \lambda ; \lambda$ is solution of (18) and $\Re \lambda>0\}$. It is well known [5] that

$$
\begin{cases}\xi_{S}\left(\omega_{j}\right)>1 & \text { if } \omega_{j}<\pi \\ \xi_{S}\left(\omega_{j}\right)>\frac{1}{2} & \text { if } \pi<\omega_{j}<2 \pi\end{cases}
$$


The singular exponents of the Laplace operator near $S_{j}$ are simpler [13]: they are equal to $\frac{(2 k+1) \pi}{2 \omega_{j}}$, with $k \in \mathbf{Z}$, if mixed boundary conditions occur near $S_{j}$ (i.e., if one has a Dirichlet boundary condition on one edge and a Neumann one on the other edge); otherwise they are equal to $\frac{k \pi}{\omega_{j}}$, with $k \in \mathbf{Z}$. The most singular positive exponent $\xi_{\Delta}\left(\omega_{j}\right)$ is then equal to (playing a similar role of $\left.\xi_{S}\left(\omega_{j}\right)\right) \frac{\pi}{2 \omega_{j}}$ in the first case and $\frac{\pi}{\omega_{j}}$ in the second.

Now we are able to state the following regularity result.

Theorem 3.1. Let $(\mathbf{u}, p, \theta) \in \mathcal{V} \times L_{0}^{2}(\Omega) \times H_{\star}^{1}(\Omega)$ be a solution of (5). Then

$$
\begin{aligned}
& (\mathbf{u}, p) \in \mathbf{H}^{2}(\Omega) \times H^{1}(\Omega), \text { if } \Omega \text { is convex, } \\
& (\mathbf{u}, p) \in \mathbf{H}^{1+s}(\Omega) \times H^{s}(\Omega), \text { if } \Omega \text { is not convex, } \\
& \theta \in H^{1+\sigma}(\Omega),
\end{aligned}
$$

where $s=\min \left(1, \min _{j=1, \ldots, n_{e}} \xi_{S}\left(\omega_{j}\right)-\varepsilon\right), \sigma=\min \left(1, \min _{j=1, \ldots, n_{e}} \xi_{\Delta}\left(\omega_{j}\right)-\varepsilon\right)$, for any $\varepsilon>0$ (except if $\min _{j=1, \ldots, n_{e}} \xi_{\Delta}\left(\omega_{j}\right)=1$, where we take $\sigma=1$ ).

Proof. As $\theta \in L^{2}(\Omega)$, we may look at $(\mathbf{u}, p)$ as solution of the Navier-Stokes equation with a datum $\mathbf{f}-\alpha \theta \in \mathbf{L}^{2}(\Omega)$ :

$$
\begin{cases}-\nu \Delta \mathbf{u}+(\mathbf{u} \cdot \nabla) \mathbf{u}+\nabla p=\mathbf{f}-\alpha \theta & \text { in } \Omega, \\ \nabla \cdot \mathbf{u}=0 & \text { in } \Omega, \\ \mathbf{u}=0 & \text { on } \partial \Omega .\end{cases}
$$

For this problem, we use the usual trick which consists in sending the nonlinear term in the right-hand side. By Theorem 1.4.4.2 of $\left[13\right.$, the fact that $\mathbf{u} \in \mathbf{H}^{1}(\Omega)$ implies that

$$
(\mathbf{u} \cdot \nabla) \mathbf{u} \in \mathbf{H}^{-\varepsilon}(\Omega) \quad \forall \varepsilon>0 .
$$

Consequently, $(\mathbf{u}, p)$ is solution of the Stokes problem with a datum $\mathbf{f}-\alpha \theta-(\mathbf{u} \cdot \nabla) \mathbf{u}$ in $\mathbf{H}^{-\varepsilon}(\Omega)$, and by Theorem 3.6 and Sections 4.1, 4.2 of [5] and the fact that $\xi_{S}\left(\omega_{j}\right)>1 / 2$, we deduce that

$$
(\mathbf{u}, p) \in \mathbf{H}^{3 / 2+\varepsilon}(\Omega) \times H^{1 / 2+\varepsilon}(\Omega),
$$

for $\varepsilon>0$ small enough. This additional regularity implies that

$$
(\mathbf{u} \cdot \nabla) \mathbf{u} \in \mathbf{L}^{2}(\Omega) .
$$

Therefore, $(\mathbf{u}, p)$ may now be seen as the solution of the Stokes problem with a datum $\mathbf{f}-\alpha \theta-(\mathbf{u} \cdot \nabla) \mathbf{u}$ in $\mathbf{L}^{2}(\Omega)$ and Theorem 3.6 and Sections 4.1, 4.2 of [5] lead to the regularities (19)-(20).

Going back to (1), we may see $\theta \in H_{\star}^{1}(\Omega)$ as solution of

$$
\begin{cases}-k \Delta \theta=g-(\mathbf{u} \cdot \nabla) \theta & \text { in } \Omega, \\ \theta=0 & \text { on } \Gamma_{D}, \\ \frac{\partial \theta}{\partial n}=0 & \text { on } \Gamma_{N} .\end{cases}
$$

By (23) and the Sobolev imbedding theorem, $\mathbf{u} \in(C(\bar{\Omega}))^{2}$, accordingly $(\mathbf{u} \cdot \nabla) \theta$ belongs to $L^{2}(\Omega)$. Therefore, $\theta$ is solution of the Laplace equation with a datum in $L^{2}(\Omega)$ and mixed boundary conditions. By Theorems 4.4.3.7 and 1.4.5.3 of [13], (21) holds (the case $\omega_{j}=\pi / 2$ with mixed conditions around $S_{j}$ is treated separately using a reflection to get the $H^{2}$-regularity). 
Corollary 3.2. If $\omega_{j}<\pi$ for all $j=1, \ldots, n_{e}$ such that mixed boundary conditions for $\theta$ occur near $S_{j}$, then

$$
\theta \in H^{3 / 2+\varepsilon}(\Omega)
$$

for $\varepsilon>0$ small enough.

Proof. It suffices to notice that the assumption implies that $\sigma>1 / 2$.

\section{A miXed FORMUlation fOR the Boussinesq EQUATIONS}

To introduce a mixed variational formulation of problem (51), we define the spaces (compare with [9, p. 118])

$$
\begin{aligned}
& \mathbf{X}=\left\{(\boldsymbol{\tau}, q) \in\left(L^{4}(\Omega)\right)^{4} \times L_{0}^{2}(\Omega),(\boldsymbol{\tau}-q \boldsymbol{\delta}) \in \mathbf{H}(\operatorname{div}, \Omega)\right\} \\
& \mathbf{Y}=\left(L^{4}(\Omega)\right)^{2} \\
& \mathbf{Z}=\left\{\eta \in\left(L^{4}(\Omega)\right)^{2} \cap H(\operatorname{div}, \Omega), \eta \cdot \mathbf{n}=0 \text { on } \Gamma_{N}\right\} \\
& T=L^{2}(\Omega)
\end{aligned}
$$

equipped with the norms

$$
\begin{gathered}
\|(\boldsymbol{\tau}, q)\|_{\mathbf{X}}=\|\boldsymbol{\tau}\|_{0,4, \Omega}+\|q\|_{0, \Omega}+\|(\boldsymbol{\tau}-q \boldsymbol{\delta})\|_{\mathbf{H}(\operatorname{div}, \Omega)}, \quad\|\mathbf{v}\|_{\mathbf{Y}}=\|\mathbf{v}\|_{0,4, \Omega}, \\
\|\eta\|_{\mathbf{z}}=\|\eta\|_{0,4, \Omega}+\|\eta\|_{H(\operatorname{div}, \Omega)}, \quad\|\psi\|_{T}=\|\psi\|_{0, \Omega} .
\end{gathered}
$$

We further introduce the following notations:

$$
(\boldsymbol{\sigma}, \boldsymbol{\tau})=\int_{\Omega} \boldsymbol{\sigma}: \boldsymbol{\tau} d x=\sum_{i, j=1}^{2} \int_{\Omega} \sigma_{i j} \tau_{i j} d x,(\mathbf{u}, \mathbf{v})=\int_{\Omega} \mathbf{u} \cdot \mathbf{v} d x=\sum_{i=1}^{2} \int_{\Omega} u_{i} v_{i} d x
$$

For a tensor $\boldsymbol{\tau}=\left(\tau_{i j}\right)_{1 \leq i, j \leq 2}$, the normal trace $\boldsymbol{\tau} \mathbf{n}$ is defined by

$$
\boldsymbol{\tau} \mathbf{n}=\left(\sum_{j=1}^{2} \tau_{1 j} n_{j}, \sum_{j=1}^{2} \tau_{2 j} n_{j}\right),
$$

where $\boldsymbol{\tau}_{i}=\left(\tau_{i 1}, \tau_{i 2}\right), i=1,2$, is a vector corresponding to the line $i$ of $\boldsymbol{\tau}$, and finally $\langle., .\rangle_{\Gamma}$ means the duality pairing between $H^{-1 / 2}(\Gamma)$ and $H^{1 / 2}(\Gamma)$.

Then the mixed formulation of (5) reads as follows. Find $(\boldsymbol{\sigma}, p) \in \mathbf{X}, \mathbf{u} \in \mathbf{Y}$, $\xi \in \mathbf{Z}$, and $\theta \in T$ solutions of (26) to (29) hereafter:

$$
\begin{aligned}
& \frac{1}{\nu}(\boldsymbol{\sigma}, \boldsymbol{\tau})+(\nabla \cdot(\boldsymbol{\tau}-q \boldsymbol{\delta}), \mathbf{u})=0 \quad \forall(\boldsymbol{\tau}, q) \in \mathbf{X}, \\
& (\nabla \cdot(\boldsymbol{\sigma}-p \boldsymbol{\delta}), \mathbf{v})-\frac{1}{\nu}(\boldsymbol{\sigma} \cdot \mathbf{u}, \mathbf{v})-(\alpha \theta, \mathbf{v})+(\mathbf{f}, \mathbf{v})=0 \quad \forall \mathbf{v} \in \mathbf{Y}, \\
& \frac{1}{k}(\xi, \eta)+(\nabla \cdot \eta, \theta)=0 \quad \forall \eta \in \mathbf{Z}, \\
& (\nabla \cdot \xi, \psi)-\frac{1}{k}(\mathbf{u} \cdot \xi, \psi)+(g, \psi)=0 \quad \forall \psi \in T .
\end{aligned}
$$

We now check that, under the assumption of Corollary [3.2, (5) is equivalent to (26)-(29): 
Theorem 4.1. Assume that $\omega_{j}<\pi$, for all $j=1, \ldots, n_{e}$, such that mixed boundary conditions occur near $S_{j}$. Then $(\mathbf{u}, \theta) \in \mathcal{V} \times H_{\star}^{1}(\Omega)$ is a solution of (5) if and only if $(\boldsymbol{\sigma}, p) \in \mathbf{X}, \mathbf{u} \in \mathbf{Y}, \xi \in \mathbf{Z}$, and $\theta \in T$ are solutions of (26) to (29), with the next relations:

$$
\begin{aligned}
\boldsymbol{\sigma} & =\nu \nabla \mathbf{u}=\nu\left(\frac{\partial u_{i}}{\partial x_{j}}\right)_{1 \leq i, j \leq 2}, \\
\xi & =k \nabla \theta .
\end{aligned}
$$

Proof. $\Rightarrow$ Let $(\mathbf{u}, \theta) \in \mathcal{V} \times H_{\star}^{1}(\Omega)$ be a solution of (15). Define $\boldsymbol{\sigma}$ and $\xi$ by (30) and (31), respectively. By Theorem 3.1 and Corollary 3.2 we have

$$
\boldsymbol{\sigma} \in\left(H^{1 / 2+\varepsilon}(\Omega)\right)^{4}, \quad \xi \in \mathbf{H}^{1 / 2+\varepsilon}(\Omega),
$$

for some $\varepsilon>0$. The Sobolev imbedding theorem yields

$$
H^{1 / 2+\varepsilon}(\Omega) \hookrightarrow L^{4}(\Omega) \quad \forall \varepsilon>0,
$$

and therefore $\sigma \in\left(L^{4}(\Omega)\right)^{4}$ and $\xi \in \mathbf{L}^{4}(\Omega)$.

Fix an arbitrary $(\boldsymbol{\tau}, q) \in \mathbf{X}$. Multiplying (30) by $\boldsymbol{\tau}$ and integrating over $\Omega$, one gets

$$
\frac{1}{\nu}(\boldsymbol{\sigma}, \boldsymbol{\tau})=(\nabla \mathbf{u}, \tau)=(\nabla \mathbf{u}, \boldsymbol{\tau}-q \boldsymbol{\delta}),
$$

because $\nabla \cdot \mathbf{u}=0$. By the following Green formula, which holds for any $\mathbf{v} \in$ $H(\operatorname{div}, \Omega)$ and any $w \in H^{1}(\Omega)$ (see the identity (I.2.17) in [12])

$$
\int_{\Omega} \nabla \cdot \mathbf{v} w d x=-\int_{\Omega} \mathbf{v} \cdot \nabla w d x+\langle\mathbf{v} \cdot \mathbf{n}, w\rangle_{\Gamma}
$$

the identity (32) becomes

$$
\frac{1}{\nu}(\boldsymbol{\sigma}, \boldsymbol{\tau})=-(\nabla \cdot(\boldsymbol{\tau}-q \boldsymbol{\delta}), \mathbf{u})+\langle(\boldsymbol{\tau}-q \boldsymbol{\delta}) \mathbf{n}, \mathbf{u}\rangle_{\Gamma} .
$$

Since $\mathbf{u}_{\mid \Gamma}=0$, we have obtained (26).

The identity (28) is proved similarly using (31).

Starting from the first identity of (5), replacing $\nabla \mathbf{u}$ by $\frac{\boldsymbol{\sigma}}{\nu}$ and using Lemma I.2.1 of [12], we have

$$
-(\boldsymbol{\sigma}-p \boldsymbol{\delta}, \nabla \mathbf{v})-\frac{1}{\nu}(\boldsymbol{\sigma} \cdot \mathbf{u}, \mathbf{v})-(\alpha \theta, \mathbf{v})+(\mathbf{f}, \mathbf{v})=0 \quad \forall \mathbf{v} \in \mathbf{H}_{0}^{1}(\Omega) .
$$

By the Green formula (33) and the fact that $\mathbf{v} \in \mathbf{H}_{0}^{1}(\Omega)$, we deduce that

$$
-(\boldsymbol{\sigma}-p \boldsymbol{\delta}, \nabla \mathbf{v})=(\nabla \cdot(\boldsymbol{\sigma}-p \boldsymbol{\delta}), \mathbf{v}) .
$$

Therefore, (27) holds for all $\mathbf{v} \in \mathbf{H}_{0}^{1}(\Omega)$ and then for all $\mathbf{v} \in \mathbf{Y}$ by density.

The identity (29) is established analogously with the help of the second identity of (5).

$\Leftarrow$ Let us fix $(\boldsymbol{\sigma}, p) \in \mathbf{X}, \mathbf{u} \in \mathbf{Y}, \xi \in \mathbf{Z}$, and $\theta \in T$ solutions of (26) to (29).

Take first as test functions in (26): $q=0$ and $\boldsymbol{\tau} \in(\mathcal{D}(\Omega))^{4}$. Then one has

$$
\frac{1}{\nu}(\boldsymbol{\sigma}, \boldsymbol{\tau})=-(\nabla \cdot \boldsymbol{\tau}, \mathbf{u}) \quad \forall \boldsymbol{\tau} \in(\mathcal{D}(\Omega))^{4},
$$

or equivalently

$$
\nabla \mathbf{u}=\frac{1}{\nu} \boldsymbol{\sigma} \text { in }\left(\mathcal{D}^{\prime}(\Omega)\right)^{4},
$$


which proves (30). Since $\mathbf{u} \in \mathbf{L}^{2}(\Omega)$ and $\boldsymbol{\sigma} \in\left(L^{2}(\Omega)\right)^{4}$, we deduce that $\mathbf{u} \in \mathbf{H}^{1}(\Omega)$. Going back to (26) with test functions $q=0$ and $\boldsymbol{\tau} \in\left(C^{\infty}(\bar{\Omega})\right)^{4}$, by the Green formula, we get

$$
\int_{\Gamma}(\boldsymbol{\tau} \mathbf{n}) \cdot \mathbf{u} d \sigma=0 \quad \forall \boldsymbol{\tau} \in\left(C^{\infty}(\bar{\Omega})\right)^{4},
$$

which implies that $\mathbf{u}_{\mid \Gamma}=0$.

Taking now in (26) $\boldsymbol{\tau}=0$ and $q \in \mathcal{D}(\Omega) \cap L_{0}^{2}(\Omega)$, we have

$$
\langle\nabla \mathbf{u}, q \boldsymbol{\delta}\rangle=0 \quad \forall q \in \mathcal{D}(\Omega) \cap L_{0}^{2}(\Omega) .
$$

Consequently, u satisfies

$$
\nabla \cdot \mathbf{u}=c \in \mathbf{C} .
$$

Applying the Green formula and the fact that $\mathbf{u}=0$ on $\Gamma$, we deduce that $\nabla \cdot \mathbf{u}=0$. This means that $\mathbf{u} \in \mathcal{V}$.

Similarly, taking appropriate test functions in (28), we show that $\theta \in H^{1}(\Omega)$, $\theta_{\mid \Gamma_{D}}=0,\left.\frac{\partial \theta}{\partial n}\right|_{\Gamma_{N}}=0$ and the identity (31).

Since $\mathcal{V}$ is a subspace of $\mathbf{Y}$, (27) implies that

$$
(\nabla \cdot(\boldsymbol{\sigma}-p \boldsymbol{\delta}), \mathbf{v})-\frac{1}{\nu}(\boldsymbol{\sigma} \cdot \mathbf{u}, \mathbf{v})-(\alpha \theta, \mathbf{v})+(\mathbf{f}, \mathbf{v})=0 \quad \forall \mathbf{v} \in \mathcal{V}
$$

Using (30) and the Green formula (33), we arrive at the first identity of (5). Remark that considering $\mathbf{v} \in(\mathcal{D}(\Omega))^{2}$, we also get $-\nu \Delta \mathbf{u}+(\mathbf{u} \cdot \nabla) \mathbf{u}+\alpha \theta+\nabla p=\mathbf{f}$ in $\left(\mathcal{D}^{\prime}(\Omega)\right)^{2}$.

The second identity of (5) follows using the trivial inclusion $H_{\star}^{1}(\Omega) \subset L^{2}(\Omega)$ and from the identity (29) combined with (31), applying Green's formula.

The Boussinesq equations (cf. [1] and Section 2) have in general more than one solution, unless the data satisfy very restrictive requirements. We propose here to analyze an approximation of nonsingular solutions of the Boussinesq mixed formulation (26)-(29) (cf. 9] and [12, pp. 298-300]). For this purpose, we define two linear operators $S$ and $L$. The operator $S$ associates to any function $\tilde{\mathbf{f}} \in$ $\left(L^{2}(\Omega)\right)^{2}$ the solution $((\tilde{\boldsymbol{\sigma}}, \tilde{p}) ; \tilde{\mathbf{u}}) \in \mathbf{X} \times \mathbf{Y}$ of the problem

$$
\begin{cases}\frac{1}{\nu}(\tilde{\boldsymbol{\sigma}}, \boldsymbol{\tau})+(\nabla \cdot(\boldsymbol{\tau}-q \boldsymbol{\delta}), \tilde{\mathbf{u}})=0 & \forall(\boldsymbol{\tau}, q) \in \mathbf{X}, \\ (\nabla \cdot(\tilde{\boldsymbol{\sigma}}-\tilde{p} \boldsymbol{\delta}), \mathbf{v})+(\tilde{\mathbf{f}}, \mathbf{v})=0 \quad \forall \mathbf{v} \in \mathbf{Y} .\end{cases}
$$

The operator $L$ associates to any function $\tilde{g} \in L^{2}(\Omega)$ the solution $(\tilde{\xi}, \tilde{\theta}) \in \mathbf{Z} \times T$ of the problem

$$
\begin{cases}\frac{1}{k}(\tilde{\xi}, \eta)+(\nabla \cdot \eta, \tilde{\theta})=0 & \forall \eta \in \mathbf{Z}, \\ (\nabla \cdot \tilde{\xi}, \psi)+(\tilde{g}, \psi)=0 & \forall \psi \in T .\end{cases}
$$

Problem (34), respectively problem (35), is nothing else than a mixed formulation of the Stokes problem (cf. [7]), respectively the Dirichlet problem, with mixed boundary conditions. Thus, using the same techniques as in [2, 7, 12] and the regularity results of Section 3 , problem (34), respectively problem (35), has a unique solution in $\mathbf{X} \times \mathbf{Y}$, respectively in $\mathbf{Z} \times T$. Furthermore, under the assumption of Corollary 3.2 , the a priori estimates

$$
\|S \tilde{\mathbf{f}}\|_{\hat{\mathbf{X}} \times \hat{\mathbf{Y}}} \leq C\|\tilde{\mathbf{f}}\|_{0, \Omega}, \quad\|L \tilde{g}\|_{\hat{\mathbf{Z}} \times T} \leq C\|\tilde{g}\|_{0, \Omega}
$$


hold with a constant $C>0$ which only depends on $\Omega$ and where

$$
\hat{\mathbf{X}}=\left(L^{r}(\Omega)\right)^{4} \times L_{0}^{2}(\Omega), \quad \hat{\mathbf{Y}}=\hat{\mathbf{Z}}=\left(L^{r}(\Omega)\right)^{2}, \quad 2<r<4
$$

and

$$
\begin{gathered}
\|((\boldsymbol{\tau}, q) ; \mathbf{u})\|_{\hat{\mathbf{X}} \times \hat{\mathbf{Y}}}=\|\boldsymbol{\tau}\|_{0, r, \Omega}+\|q\|_{0, \Omega}+\|\mathbf{u}\|_{0, r, \Omega}, \\
\|(\eta, \psi)\|_{\hat{\mathbf{Z}} \times T}=\|\eta\|_{0, r, \Omega}+\|\psi\|_{0, \Omega} .
\end{gathered}
$$

Note that we have the following continuous injections:

$$
\mathbf{X} \hookrightarrow \hat{\mathbf{X}}, \quad \mathbf{Y} \hookrightarrow \hat{\mathbf{Y}}, \quad \mathbf{Z} \hookrightarrow \hat{\mathbf{Z}}
$$

Next we define the mapping $H$ from $\underset{\sim}{\mathbf{X}} \times \underset{\sim}{\mathbf{Z}}$ into itself by

$$
H(\underset{\sim}{\boldsymbol{\tau}}, \underset{\sim}{\eta})=\left(\begin{array}{c}
\underset{\boldsymbol{\tau}}{\sim} \\
\underset{\sim}{\eta}
\end{array}\right)-\left(\begin{array}{ll}
S & 0 \\
0 & L
\end{array}\right)\left(\begin{array}{c}
\mathbf{f}-\frac{1}{\nu}(\boldsymbol{\tau} \cdot \mathbf{v})-\alpha \psi \\
g-\frac{1}{k}(\mathbf{v} \cdot \eta)
\end{array}\right)
$$

where $\underset{\sim}{\mathbf{X}}=\mathbf{X} \times \mathbf{Y}, \underset{\sim}{\mathbf{Z}}=\mathbf{Z} \times T, \underset{\sim}{\boldsymbol{\tau}}=((\boldsymbol{\tau}, q) ; \mathbf{v})$, and $\underset{\sim}{\eta}=(\eta, \psi)$.

With these notations the Boussinesq equations (26)-(29) take the form

$$
\text { Find } \underset{\sim}{\boldsymbol{\sigma}}, \underset{\sim}{\xi}) \in \underset{\sim}{\mathbf{X}} \times \underset{\sim}{\mathbf{Z}} \text { such that } H(\underset{\sim}{\boldsymbol{\sigma}}, \underset{\sim}{\xi})=0
$$

where $\underset{\sim}{\boldsymbol{\sigma}}=((\boldsymbol{\sigma}, p) ; \mathbf{u})$ and $\xi=(\xi, \theta)$.

In the sequel, we shall be concerned with the nonsingular solution of (37). A solution $(\underset{\sim}{\boldsymbol{\sigma}}, \underset{\sim}{\xi}) \in \underset{\sim}{\mathbf{X}} \times \underset{\sim}{\mathbf{Z}}$ of $(37)$ is said to be nonsingular if the Frechet derivative of $H$ at the point $(\underset{\sim}{\boldsymbol{\sigma}}, \xi): H^{\prime}(\underset{\sim}{\boldsymbol{\sigma}}, \xi): \underset{\sim}{\mathbf{X}} \times \underset{\sim}{\mathbf{Z}} \longrightarrow \underset{\sim}{\mathbf{X}} \times \underset{\sim}{\mathbf{Z}}$

$$
H^{\prime}(\underset{\sim}{\boldsymbol{\sigma}}, \underset{\sim}{\xi})(\underset{\sim}{\boldsymbol{\tau}}, \underset{\sim}{\eta})=\left(\begin{array}{c}
\underset{\sim}{\sim} \\
\underset{\sim}{\eta}
\end{array}\right)+\left(\begin{array}{cc}
S & 0 \\
0 & L
\end{array}\right)\left(\begin{array}{c}
\frac{1}{\nu}(\boldsymbol{\sigma} \cdot \mathbf{v}+\boldsymbol{\tau} \cdot \mathbf{u})+\alpha \psi \\
\frac{1}{k}(\mathbf{u} \cdot \eta+\mathbf{v} \cdot \xi)
\end{array}\right)
$$

is an isomorphism

Hence, $\underset{\sim}{\boldsymbol{\sigma}}, \xi)$ is a nonsingular solution of (37) if and only if, for each $\mathbf{f}^{*} \in$ $\left(L^{2}(\Omega)\right)^{2}$ and $\tilde{g}^{*} \in L^{2}(\Omega)$, the linearized Boussinesq problem

$$
\text { Find } \quad\left(\underset{\sim}{\boldsymbol{\sigma}}, \underset{\sim}{\xi^{*}}\right) \in \underset{\sim}{\mathbf{X}} \times \underset{\sim}{\mathbf{Z}} \text { such that } H^{\prime}(\underset{\sim}{\boldsymbol{\sigma}}, \underset{\sim}{\xi})\left(\underset{\sim}{\boldsymbol{\sigma}}, \underset{\sim}{\xi^{*}}\right)=\left(\begin{array}{cc}
S & 0 \\
0 & L
\end{array}\right)\left(\begin{array}{l}
\mathbf{f}^{*} \\
g^{*}
\end{array}\right)
$$

is well posed.

Now in order to study the nonsingular solution of (37) we introduce the bounded linear operator

$$
\left\{\begin{array}{l}
\mathcal{K} \in \underset{\sim}{\mathcal{L}}(\underset{\sim}{\mathbf{X}} \times \underset{\sim}{\mathbf{Z}}, \underset{\sim}{\mathbf{X}} \times \underset{\sim}{\mathbf{Z}}) \\
\mathcal{K}(\underset{\sim}{\boldsymbol{\tau}}, \underset{\sim}{\eta})=\left(\begin{array}{cc}
S & 0 \\
0 & L
\end{array}\right)\left(\begin{array}{c}
\frac{1}{\nu}(\boldsymbol{\sigma} \cdot \mathbf{v}+\boldsymbol{\tau} \cdot \mathbf{u})+\alpha \psi \\
\frac{1}{k}(\mathbf{u} \cdot \eta+\mathbf{v} \cdot \xi)
\end{array}\right),
\end{array}\right.
$$

and we state the following result.

Lemma 4.2. Assume that $\underset{\sim}{\boldsymbol{\sigma}}, \underset{\sim}{\xi}) \in \underset{\sim}{\mathbf{X}} \times \underset{\sim}{\mathbf{Z}}$ is a nonsingular solution of (37) such that $\mathbf{u} \in \mathbf{H}^{2}(\Omega)$ and $\theta \in H^{2}(\Omega)$. Then the operator $(I+\mathcal{K})$ is invertible and has a continuous inverse in $\underset{\sim}{\mathcal{L}}(\underset{\sim}{\hat{\mathbf{X}}} \times \underset{\sim}{\hat{\mathbf{Z}}}, \underset{\sim}{\hat{\mathbf{X}}} \times \underset{\sim}{\hat{\mathbf{Z}}})$, where $\underset{\sim}{\hat{\mathbf{X}}}=\hat{\mathbf{X}} \times \hat{\mathbf{Y}}$ and $\underset{\sim}{\hat{\mathbf{Z}}}=\hat{\mathbf{Z}} \times T$.

The proof of this lemma is similar to the one of Lemma 2.1 in [9]. 


\section{THE DISCRETE PROBLEM AND ERROR ESTIMATES}

Let us now introduce the discrete version of (37) by using mixed finite element methods.

Let $\left(\mathcal{T}_{h}\right)_{h>0}$ be a uniformly regular (or quasi-uniform) family of triangulations of $\Omega$ (see [3] or [12 p. 98]), in the sense that there exist two positive constants $\sigma, \tau$ such that

$$
\tau h \leq h_{K} \leq \sigma \rho_{K} \quad \forall K \in \mathcal{T}_{h} \forall h>0,
$$

where $h_{K}$ (resp. $\rho_{K}$ ) denotes the exterior (resp. interior) diameter of $K$. For $K \subset \mathbb{R}^{2}$, let $P_{k}, k \geq 0$ denote the restrictions of polynomials of total degree $\leq k$ to $K$.

For any $K \in \mathcal{T}_{h}$ and $\mathbf{x}=\left(x_{1}, x_{2}\right)$, let

$$
R T_{0}=\left(P_{0}\right)^{2} \oplus \mathbf{x} P_{0}=\left\{(a, b)+c\left(x_{1}, x_{2}\right) ; a, b, c \in \mathbb{R}\right\}
$$

and set

$$
\begin{aligned}
& \mathbf{X}_{h}=\left\{\left(\boldsymbol{\tau}_{h}, q_{h}\right) \in \mathbf{X} ; \boldsymbol{\tau}_{h \mid K} \in\left(R T_{0}\right)^{2}, q_{h \mid K} \in P_{0} \forall K \in \mathcal{T}_{h}\right\}, \\
& \mathbf{Y}_{h}=\left\{\mathbf{v}_{h} \in \mathbf{Y} ; \mathbf{v}_{h \mid K} \in\left(P_{0}\right)^{2} \forall K \in \mathcal{T}_{h}\right\}, \\
& \mathbf{Z}_{h}=\left\{\eta_{h} \in \mathbf{Z} ; \eta_{h \mid K} \in R T_{0} \forall K \in \mathcal{T}_{h}\right\}, \\
& T_{h}=\left\{\psi_{h} \in T ; \psi_{h \mid K} \in P_{0} \forall K \in \mathcal{T}_{h}\right\} .
\end{aligned}
$$

Observe that the definition of $\mathbf{Z}_{h}$ is possible if the partition into elements is made in such a way that there is no element across the interface between $\Gamma_{D}$ and $\Gamma_{N}$ on $\Gamma$.

We have the following approximation results (cf. [3, 12, 15]):

i) Owing to Theorem III.4.4 of [12], there exist two interpolant operators $\Pi_{h} \in$ $\mathcal{L}\left(\mathbf{X} \cap\left(\left(\mathbf{H}^{1}(\Omega)\right)^{2} \times H^{1}(\Omega)\right), \mathbf{X}_{h}\right)$ and $\Pi_{h}^{0} \in \mathcal{L}\left(\mathbf{Z} \cap\left(H^{1}(\Omega)\right)^{2}, \mathbf{Z}_{h}\right)$ such that

$$
\begin{aligned}
& \left\|\left(\Pi_{h}-I\right)(\boldsymbol{\tau}, q)\right\|_{0, s, \Omega} \leq C h^{2 / s}|(\boldsymbol{\tau}, q)|_{1, \Omega} \quad \forall s \geq 2, \\
& \left\|\Pi_{h}^{0} \eta-\eta\right\|_{0, s, \Omega} \leq C h^{2 / s}|\eta|_{1, \Omega} \quad \forall s \geq 2,
\end{aligned}
$$

where $|(\boldsymbol{\tau}, q)|_{1, \Omega}=|\boldsymbol{\tau}|_{1, \Omega}+|q|_{1, \Omega}$ and $C$ is a positive constant independent of $h$;

ii) Owing to Theorem 3.1.5 of [3], there exist two projection operators $\mathcal{P}_{h} \in$ $\mathcal{L}\left(\mathbf{H}^{1}(\Omega), \mathbf{Y}_{h}\right)$ and $\mathcal{P}_{h}^{0} \in \mathcal{L}\left(H^{1}(\Omega), T_{h}\right)$ such that

$$
\begin{array}{ll}
\left\|\mathcal{P}_{h} \mathbf{v}-\mathbf{v}\right\|_{0, s, \Omega} \leq C h^{2 / s}|\mathbf{v}|_{1, \Omega} \quad & \forall s \geq 2 \\
\left\|\mathcal{P}_{h}^{0} \psi-\psi\right\|_{0, s, \Omega} \leq C h^{2 / s}|\psi|_{1, \Omega} & \forall s \geq 2
\end{array}
$$

Now, in order to write the discrete problem in the same form as the continuous problem, we introduce the discrete operators $S_{h}$ and $L_{h}$ of $S$ and $L$. For $\tilde{\mathbf{f}} \in$ $\left(L^{2}(\Omega)\right)^{2}, S_{h} \tilde{\mathbf{f}}=\left(\left(\tilde{\boldsymbol{\sigma}}_{h}, \tilde{p}_{h}\right) ; \tilde{\mathbf{u}}_{h}\right) \in \mathbf{X}_{h} \times \mathbf{Y}_{h}$ is the solution of the problem

$$
\left\{\begin{array}{l}
\frac{1}{\nu}\left(\tilde{\boldsymbol{\sigma}}_{h}, \boldsymbol{\tau}_{h}\right)+\left(\nabla \cdot\left(\boldsymbol{\tau}_{h}-q_{h} \boldsymbol{\delta}\right), \tilde{\mathbf{u}}_{h}\right)=0 \quad \forall\left(\boldsymbol{\tau}_{h}, q_{h}\right) \in \mathbf{X}_{h} \\
\left(\nabla \cdot\left(\tilde{\boldsymbol{\sigma}}_{h}-\tilde{p}_{h} \boldsymbol{\delta}\right), \mathbf{v}_{h}\right)+\left(\tilde{\mathbf{f}}, \mathbf{v}_{h}\right)=0 \quad \forall \mathbf{v}_{h} \in \mathbf{Y}_{h} .
\end{array}\right.
$$


On the other hand, for any $\tilde{g} \in L^{2}(\Omega), L_{h} \tilde{g}=\left(\tilde{\xi}_{h}, \tilde{\theta}_{h}\right) \in \mathbf{Z}_{h} \times T_{h}$ is the solution of the problem

$$
\left\{\begin{array}{l}
\frac{1}{k}\left(\tilde{\xi}_{h}, \eta_{h}\right)+\left(\nabla \cdot \eta_{h}, \tilde{\theta}_{h}\right)=0 \quad \forall \eta_{h} \in \mathbf{Z}_{h} \\
\left(\nabla \cdot \tilde{\xi}_{h}, \psi_{h}\right)+\left(\tilde{g}, \psi_{h}\right)=0 \quad \forall \psi_{h} \in T_{h} .
\end{array}\right.
$$

Next we define the mapping $H_{h}$ from $\underset{\sim}{\mathbf{X}_{h}} \times \underset{\sim}{\mathbf{Z}_{h}}$ into itself by

$$
H_{h}\left(\underset{\sim}{\boldsymbol{\tau}_{h}, \eta_{h}}\right)=\left(\begin{array}{c}
\boldsymbol{\tau}_{\sim} \\
\underset{\sim}{\eta_{h}}
\end{array}\right)-\left(\begin{array}{cc}
S_{h} & 0 \\
0 & L_{h}
\end{array}\right)\left(\begin{array}{c}
\mathbf{f}-\frac{1}{\nu}\left(\boldsymbol{\tau}_{h} \cdot \mathbf{v}_{h}\right)-\alpha \psi_{h} \\
g-\frac{1}{k}\left(\mathbf{v}_{h} \cdot \eta_{h}\right)
\end{array}\right),
$$

where $\underset{\sim}{\mathbf{X}_{h}}=\mathbf{X}_{h} \times \mathbf{Y}_{h}, \underset{\sim}{\mathbf{Z}_{h}}=\mathbf{Z}_{h} \times T_{h}, \underset{\sim}{\tau_{h}}=\left(\left(\boldsymbol{\tau}_{h}, q_{h}\right) ; \mathbf{v}_{h}\right)$, and $\underset{\sim}{\eta_{h}}=\left(\eta_{h}, \psi_{h}\right)$.

Then, the discrete problem of (37) reads as follows:

$$
\left\{\begin{array}{l}
\text { Find } \underset{\sim}{\left(\boldsymbol{\sigma}_{h}, \xi_{h}\right) \in \underset{\sim}{\mathbf{X}_{h}} \times \underset{\sim}{\mathbf{Z}_{h}} \text { such that }} \\
H_{h}\left(\underset{\sim}{\left.\boldsymbol{\sigma}_{h}, \xi_{h}\right)}=0\right.
\end{array}\right.
$$

where $\boldsymbol{\sigma}_{\sim}=\left(\left(\boldsymbol{\sigma}_{h}, p_{h}\right) ; \mathbf{u}_{h}\right)$ and $\underset{\sim}{\xi_{h}}=\left(\xi_{h}, \theta_{h}\right)$.

Finally, we introduce the discrete operator $\mathcal{K}_{h}$ of $\mathcal{K}$. Let $((\boldsymbol{\sigma}, p) ; u) \in \mathbf{X} \times \mathbf{Y}$, $(\xi, \theta) \in \mathbf{Z} \times T$ and set $\left(\left(\boldsymbol{\sigma}_{h}^{*}, p_{h}^{*}\right) ; \mathbf{u}_{h}^{*}\right)=\left(\Pi_{h}(\boldsymbol{\sigma}, p) ; \mathcal{P}_{h} \mathbf{u}\right),\left(\xi_{h}^{*}, \theta_{h}^{*}\right)=\left(\Pi_{h}^{0} \xi, \mathcal{P}_{h}^{0} \theta\right)$. The

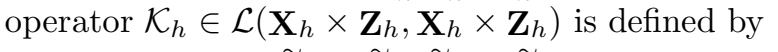

$$
\mathcal{K}_{h}\left(\underset{\sim}{\boldsymbol{\tau}_{h},} \underset{\sim}{\eta_{h}}\right)=\left(\begin{array}{cc}
S_{h} & 0 \\
0 & L_{h}
\end{array}\right)\left(\begin{array}{c}
\frac{1}{\nu}\left(\boldsymbol{\sigma}_{h}^{*} \cdot \mathbf{v}_{h}+\boldsymbol{\tau}_{h} \cdot \mathbf{u}_{h}^{*}\right)+\alpha \psi_{h} \\
\frac{1}{k}\left(\mathbf{u}_{h}^{*} \cdot \eta_{h}+\mathbf{v}_{h} \cdot \xi_{h}^{*}\right)
\end{array}\right),
$$

where $\boldsymbol{\tau}_{\sim}=\left(\left(\boldsymbol{\tau}_{h}, q_{h}\right) ; \mathbf{v}_{h}\right) \in \underset{\sim}{\mathbf{X}_{h}}$ and $\underset{\sim}{\eta_{h}}=\left(\eta_{h}, \psi_{h}\right) \in \underset{\sim}{\mathbf{Z}_{h}}$.

We now prove some technical lemmas.

Lemma 5.1. For all $r$ such that $2<r<4$, we have

$$
\begin{gathered}
\sup _{\left(\boldsymbol{\tau}_{h}, q_{h}\right) \in \mathbf{X}_{h}} \frac{\left(\nabla \cdot\left(\boldsymbol{\tau}_{h}-q_{h} \boldsymbol{\delta}\right), \mathbf{v}_{h}\right)}{\left\|\left(\boldsymbol{\tau}_{h}, q_{h}\right)\right\|_{\hat{\mathbf{X}}}} \geq C\left\|\mathbf{v}_{h}\right\|_{0, r, \Omega} \quad \forall \mathbf{v}_{h} \in \mathbf{Y}_{h}, \\
\sup _{\eta_{h} \in \mathbf{Z}_{h}} \frac{\left(\nabla \cdot \eta_{h}, \psi_{h}\right)}{\left\|\eta_{h}\right\|_{\hat{\mathbf{z}}}} \geq C\left\|\psi_{h}\right\|_{0, \Omega} \quad \forall \psi_{h} \in T_{h},
\end{gathered}
$$

where $\|(\boldsymbol{\tau}, q)\|_{\hat{\mathbf{X}}}=\|\boldsymbol{\tau}\|_{0, r, \Omega}+\|q\|_{0, \Omega},\|\eta\|_{\hat{\mathbf{z}}}=\|\eta\|_{0, r, \Omega}$ and $C$ is a positive constant independent of $h$.

Proof. Let us sketch the proof of (50). For any $\mathbf{v}_{h} \in \mathbf{Y}_{h}$, there exists (cf. 12, p. 255]) $\boldsymbol{\tau}_{h} \in\left\{\boldsymbol{\tau} \in \mathbf{H}(\operatorname{div}, \Omega), \boldsymbol{\tau}_{\mid K} \in\left(R T_{0}\right)^{2} \forall K \in \mathcal{T}_{h}\right\}$ such that $\left(\nabla \cdot \boldsymbol{\tau}_{h}, \mathbf{v}_{h}\right)=\left\|\mathbf{v}_{h}\right\|_{0, \Omega}^{2}$ and $\left\|\boldsymbol{\tau}_{h}\right\|_{0, s, \Omega}+\left\|\nabla \cdot \boldsymbol{\tau}_{h}\right\|_{0, \Omega} \leq C\left\|\mathbf{v}_{h}\right\|_{0, \Omega} \forall s \geq 2$. Now $\left(\boldsymbol{\tau}_{h}, 0\right) \in \mathbf{X}_{h}$ and from the direct and inverse estimates

$$
\left\|\boldsymbol{\tau}_{h}\right\|_{0, r, \Omega} \leq C h^{\frac{2}{r}-\frac{2}{s}}\left\|\boldsymbol{\tau}_{h}\right\|_{0, s, \Omega}, \quad\left\|\mathbf{v}_{h}\right\|_{0, r, \Omega} \leq C h^{\frac{2}{r}-1}\left\|\mathbf{v}_{h}\right\|_{0, \Omega},
$$

we have

$$
\frac{\left(\nabla \cdot \boldsymbol{\tau}_{h}, \mathbf{v}_{h}\right)}{\left\|\left(\boldsymbol{\tau}_{h}, 0\right)\right\|_{\hat{\mathbf{X}}}}=\frac{\left\|\mathbf{v}_{h}\right\|_{0, \Omega}^{2}}{\left\|\boldsymbol{\tau}_{h}\right\|_{0, r, \Omega}} \geq C h^{1+\frac{2}{s}-\frac{4}{r}}\left\|\mathbf{v}_{h}\right\|_{0, r, \Omega}
$$


Therefore, taking $s=2 r /(4-r)(s>2$ since $2<r<4)$, we obtain

$$
\frac{\left(\nabla \cdot \boldsymbol{\tau}_{h}, \mathbf{v}_{h}\right)}{\left\|\left(\boldsymbol{\tau}_{h}, 0\right)\right\|_{\hat{\mathbf{X}}}} \geq C\left\|\mathbf{v}_{h}\right\|_{0, r, \Omega}
$$

and (50) follows immediately.

Lemma 5.2. Assume that $\Omega$ is convex and that $\omega_{j} \leq \frac{\pi}{2}$, for all $j=1, \ldots, n_{e}$, such that mixed boundary conditions for $\theta$ occur near $S_{j}$. Let $\tilde{\mathbf{f}} \in\left(L^{2}(\Omega)\right)^{2}$ and $\tilde{g} \in L^{2}(\Omega)$. Then each of the problems (45) and [46) has a unique solution and

$$
\begin{gathered}
\left\|\left(S_{h}-S\right) \tilde{\mathbf{f}}\right\|_{\hat{\mathbf{X}} \times \hat{\mathbf{Y}}} \leq C h^{2 / r}\|S \tilde{\mathbf{f}}\|_{1, \Omega}, \\
\left\|\left(L_{h}-L\right) \tilde{g}\right\|_{\hat{\mathbf{z}} \times T} \leq C h^{2 / r}\|L \tilde{g}\|_{1, \Omega}
\end{gathered}
$$

Proof. Owing to the inf-sup conditions (50) and (51), it is a routine matter to show that each of the problems (45) and (46) has a unique solution.

To prove the estimate $(52)$, let $((\tilde{\boldsymbol{\sigma}}, \tilde{p}) ; \tilde{\mathbf{u}})=S \tilde{\mathbf{f}},\left(\left(\tilde{\boldsymbol{\sigma}}_{h}, \tilde{p}_{h}\right) ; \tilde{\mathbf{u}}_{h}\right)=S_{h} \tilde{\mathbf{f}}$ and set $\left(\left(\tilde{\boldsymbol{\sigma}}_{h}^{*}, \tilde{p}_{h}^{*}\right) ; \tilde{\mathbf{u}}_{h}^{*}\right)=\left(\Pi_{h}(\tilde{\boldsymbol{\sigma}}, \tilde{p}) ; \mathcal{P}_{h} \tilde{\mathbf{u}}\right)$. Now, similarly to Theorem 3.2 of [8], using the theory of mixed finite element methods (cf. [2]) and the fact that (cf. [7])

$$
\begin{gathered}
\frac{1}{\nu}\left\|\boldsymbol{\tau}_{h}\right\|_{0, \Omega}^{2} \geq C\left\|\left(\boldsymbol{\tau}_{h}, q_{h}\right)\right\|_{0, \Omega}^{2} \\
\forall\left(\boldsymbol{\tau}_{h}, q_{h}\right) \in\left\{(\boldsymbol{\tau}, q) \in \mathbf{X}_{h} ;(\nabla \cdot(\boldsymbol{\tau}-q \boldsymbol{\delta}), \mathbf{v})=0 \forall \mathbf{v} \in \mathbf{Y}_{h}\right\},
\end{gathered}
$$

we have

$$
\left\|\tilde{\boldsymbol{\sigma}}_{h}^{*}-\tilde{\boldsymbol{\sigma}}_{h}\right\|_{0, \Omega}+\left\|\tilde{p}_{h}^{*}-\tilde{p}_{h}\right\|_{0, \Omega}+\left\|\tilde{\mathbf{u}}_{h}^{*}-\tilde{\mathbf{u}}_{h}\right\|_{0, \Omega} \leq C h\left(|\tilde{\boldsymbol{\sigma}}|_{1, \Omega}+|\tilde{p}|_{1, \Omega}+|\tilde{\mathbf{u}}|_{1, \Omega}\right) .
$$

On the other hand, since (cf. [3, 6])

$$
\begin{gathered}
\left\|\tilde{\boldsymbol{\sigma}}_{h}^{*}-\tilde{\boldsymbol{\sigma}}_{h}\right\|_{0, r, \Omega} \leq C h^{2 / r-1}\left\|\tilde{\boldsymbol{\sigma}}_{h}^{*}-\tilde{\boldsymbol{\sigma}}_{h}\right\|_{0, \Omega} ; \\
\left\|\tilde{\mathbf{u}}_{h}^{*}-\tilde{\mathbf{u}}_{h}\right\|_{0, r, \Omega} \leq C h^{2 / r-1}\left\|\tilde{\mathbf{u}}_{h}^{*}-\tilde{\mathbf{u}}_{h}\right\|_{0, \Omega},
\end{gathered}
$$

and $r>2$, the estimate (54) leads to

$$
\begin{gathered}
\left\|\tilde{\boldsymbol{\sigma}}_{h}^{*}-\tilde{\boldsymbol{\sigma}}_{h}\right\|_{0, r, \Omega}+\left\|\tilde{p}_{h}^{*}-\tilde{p}_{h}\right\|_{0, \Omega}+\left\|\tilde{\mathbf{u}}_{h}^{*}-\tilde{\mathbf{u}}_{h}\right\|_{0, r, \Omega} \\
\leq C h^{2 / r}\left(|\tilde{\boldsymbol{\sigma}}|_{1, \Omega}+|\tilde{p}|_{1, \Omega}+|\tilde{\mathbf{u}}|_{1, \Omega}\right) .
\end{gathered}
$$

This last inequality, with (41) and (43), gives us

$$
\begin{array}{r}
\left\|\tilde{\boldsymbol{\sigma}}-\tilde{\boldsymbol{\sigma}}_{h}\right\|_{0, r, \Omega}+\left\|\tilde{p}-\tilde{p}_{h}\right\|_{0, \Omega}+\left\|\tilde{\mathbf{u}}-\tilde{\mathbf{u}}_{h}\right\|_{0, r, \Omega} \\
\leq C h^{2 / r}\left(|\tilde{\boldsymbol{\sigma}}|_{1, \Omega}+|\tilde{p}|_{1, \Omega}+|\tilde{\mathbf{u}}|_{1, \Omega}\right),
\end{array}
$$

which is nothing else than (52).

The proof of (53) is similar to the one above.

Remark 5.3. From (52) and (53) one can deduce the following estimates

$$
\begin{array}{ll}
\left\|\left(S_{h}-S\right) \tilde{\mathbf{f}}\right\|_{\hat{\mathbf{X}} \times \hat{\mathbf{Y}}} \leq C h^{2 / r}\|\tilde{\mathbf{f}}\|_{0, \Omega} & \forall \tilde{\mathbf{f}} \in\left(L^{2}(\Omega)\right)^{2}, \\
\left\|\left(L_{h}-L\right) \tilde{g}\right\|_{\hat{\mathbf{Z}} \times T} \leq C h^{2 / r}\|\tilde{g}\|_{0, \Omega} & \forall \tilde{g} \in L^{2}(\Omega) .
\end{array}
$$

Lemma 5.4. Under the assumptions on $\Omega$ of Lemma 5.2, assume that $\underset{\sim}{\boldsymbol{\sigma}}, \boldsymbol{\sim}$ ) is a nonsingular solution of (37). Then we have




Proof. Remark first that the assumptions on $\Omega$ of Lemma 5.2 yield with Theorem $3.1 \mathbf{u} \in \mathbf{H}^{2}(\Omega)$ and $\theta \in H^{2}(\Omega)$.

Let $(\underset{\sim}{\boldsymbol{\tau}}, \eta) \in \underset{\sim}{\boldsymbol{X}} \times \underset{\sim}{\mathbf{Z}},\left(\left(\boldsymbol{\sigma}_{h}^{*}, p_{h}^{*}\right) ; \mathbf{u}_{h}^{*}\right)=\left(\Pi_{h}(\boldsymbol{\sigma}, p) ; \mathcal{P}_{h} \mathbf{u}\right)$ and $\left(\xi_{h}^{*}, \theta_{h}^{*}\right)=\left(\Pi_{h}^{0} \xi, \mathcal{P}_{h}^{0} \theta\right)$.

We have

$$
\begin{aligned}
\left(\mathcal{K}-\mathcal{K}_{h}\right)(\underset{\sim}{\boldsymbol{\tau}}, \underset{\sim}{\eta})= & \left(\begin{array}{c}
\frac{1}{\nu}\left(S(\boldsymbol{\sigma} \cdot \mathbf{v}+\boldsymbol{\tau} \cdot \mathbf{u})-S_{h}\left(\boldsymbol{\sigma}_{h}^{*} \cdot \mathbf{v}+\boldsymbol{\tau} \cdot \mathbf{u}_{h}^{*}\right)\right) \\
\frac{1}{k}\left(L(\mathbf{u} \cdot \eta+\mathbf{v} \cdot \xi)-L_{h}\left(\mathbf{u}_{h}^{*} \cdot \eta+\mathbf{v} \cdot \xi_{h}^{*}\right)\right)
\end{array}\right) \\
& +\left(\begin{array}{c}
\left(S-S_{h}\right)(\alpha \psi) \\
0
\end{array}\right) .
\end{aligned}
$$

In order to estimate $\left(\mathcal{K}-\mathcal{K}_{h}\right)(\underset{\sim}{\boldsymbol{\tau}}, \underset{\sim}{\eta})$, we shall estimate each term of the right-hand side of (58). First, owing to (55), we have

$$
\left\|\left(S-S_{h}\right)(\alpha \psi)\right\|_{\hat{\mathbf{X}} \times \hat{\mathbf{Y}}} \leq C h^{2 / r}\|\alpha \psi\|_{0, \Omega} \leq C(\alpha) h^{2 / r}\|\psi\|_{0, \Omega} .
$$

The term $S(\boldsymbol{\sigma} \cdot \mathbf{v}+\boldsymbol{\tau} \cdot \mathbf{u})-S_{h}\left(\boldsymbol{\sigma}_{h}^{*} \cdot \mathbf{v}+\boldsymbol{\tau} \cdot \mathbf{u}_{h}^{*}\right)$ can be written as follows

$$
\begin{aligned}
& S(\boldsymbol{\sigma} \cdot \mathbf{v}+\boldsymbol{\tau} \cdot \mathbf{u})-S_{h}\left(\boldsymbol{\sigma}_{h}^{*} \cdot \mathbf{v}+\boldsymbol{\tau} \cdot \mathbf{u}_{h}^{*}\right) \\
& \quad=S\left(\left(\boldsymbol{\sigma}-\boldsymbol{\sigma}_{h}^{*}\right) \cdot \mathbf{v}\right)+S\left(\boldsymbol{\tau} \cdot\left(\mathbf{u}-\mathbf{u}_{h}^{*}\right)\right)+\left(S-S_{h}\right)\left(\boldsymbol{\sigma}_{h}^{*} \cdot \mathbf{v}\right)+\left(S-S_{h}\right)\left(\boldsymbol{\tau} \cdot \mathbf{u}_{h}^{*}\right) .
\end{aligned}
$$

Using the fact that $\|S \tilde{\mathbf{f}}\|_{\hat{\mathbf{X}} \times \hat{\mathbf{Y}}} \leq C\|\tilde{\mathbf{f}}\|_{0, \Omega}$ for all $\tilde{\mathbf{f}} \in\left(L^{2}(\Omega)\right)^{2}$, (41), (43) and (55), we get

$$
\begin{aligned}
\left\|S\left(\left(\boldsymbol{\sigma}-\boldsymbol{\sigma}_{h}^{*}\right) \cdot \mathbf{v}\right)\right\|_{\hat{\mathbf{X}} \times \hat{\mathbf{Y}}} & \leq C\left\|\left(\boldsymbol{\sigma}-\boldsymbol{\sigma}_{h}^{*}\right) \cdot \mathbf{v}\right\|_{0, \Omega} \\
& \leq C\left\|\boldsymbol{\sigma}-\boldsymbol{\sigma}_{h}^{*}\right\|_{0, s, \Omega}\|\mathbf{v}\|_{0, r, \Omega} \\
& \leq C h^{2 / s}|(\boldsymbol{\sigma}, p)|_{1, \Omega}\|\mathbf{v}\|_{0, r, \Omega}
\end{aligned}
$$

where $s=2 r /(r-2)>2$ since $r>2$,

$$
\begin{aligned}
\left\|S\left(\boldsymbol{\tau} \cdot\left(\mathbf{u}-\mathbf{u}_{h}^{*}\right)\right)\right\|_{\hat{\mathbf{X}} \times \hat{\mathbf{Y}}} & \leq C\left\|\boldsymbol{\tau} \cdot\left(\mathbf{u}-\mathbf{u}_{h}^{*}\right)\right\|_{0, \Omega} \\
& \leq C\|\boldsymbol{\tau}\|_{0, r, \Omega}\left\|\mathbf{u}-\mathbf{u}_{h}^{*}\right\|_{0, s, \Omega} \\
& \leq C h^{2 / s}|\mathbf{u}|_{1, \Omega}\|\boldsymbol{\tau}\|_{0, r, \Omega}, \\
\left\|\left(S-S_{h}\right)\left(\boldsymbol{\sigma}_{h}^{*} \cdot \mathbf{v}\right)\right\|_{\hat{\mathbf{x}} \times \hat{\mathbf{Y}}} & \leq C h^{2 / r}\left\|\boldsymbol{\sigma}_{h}^{*} \cdot \mathbf{v}\right\|_{0, \Omega} \\
& \leq C h^{2 / r}\left\|\boldsymbol{\sigma}_{h}^{*}\right\|_{0, s, \Omega}\|\mathbf{v}\|_{0, r, \Omega} \\
& \leq C h^{2 / r}\|(\boldsymbol{\sigma}, p)\|_{1, \Omega}\|\mathbf{v}\|_{0, r, \Omega}, \\
\left\|\left(S-S_{h}\right)\left(\boldsymbol{\tau} \cdot \mathbf{u}_{h}^{*}\right)\right\|_{\hat{\mathbf{x}} \times \hat{\mathbf{Y}}} & \leq C h^{2 / r}\left\|\boldsymbol{\tau} \cdot \mathbf{u}_{h}^{*}\right\|_{0, \Omega} \\
& \leq C h^{2 / r}\|\boldsymbol{\tau}\|_{0, r, \Omega}\left\|\mathbf{u}_{h}^{*}\right\|_{0, s, \Omega} \\
& \leq C h^{2 / r}\|\mathbf{u}\|_{1, \Omega}\|\boldsymbol{\tau}\|_{0, r, \Omega} .
\end{aligned}
$$

Therefore, using these estimates and (601), we have

$$
\left\|S(\boldsymbol{\sigma} \cdot \mathbf{v}+\boldsymbol{\tau} \cdot \mathbf{u})-S_{h}\left(\boldsymbol{\sigma}_{h}^{*} \cdot \mathbf{v}+\boldsymbol{\tau} \cdot \mathbf{u}_{h}^{*}\right)\right\|_{\hat{\mathbf{X}} \times \hat{\mathbf{Y}}} \leq C h^{\min (2 / r, 2 / s)}\left(\|\boldsymbol{\tau}\|_{0, r, \Omega}+\|\mathbf{v}\|_{0, r, \Omega}\right) .
$$


A similar procedure leads to

$$
\left\|L(\mathbf{u} \cdot \eta+\mathbf{v} \cdot \xi)-L_{h}\left(\mathbf{u}_{h}^{*} \cdot \eta+\mathbf{v} \cdot \xi_{h}^{*}\right)\right\|_{\hat{\mathbf{z}} \times T} \leq C h^{\min (2 / r, 2 / s)}\left(\|\eta\|_{0, r, \Omega}+\|\mathbf{v}\|_{0, r, \Omega}\right) .
$$

We then get, from (58), (59), (61) and (62),

$$
\left\|\left(\mathcal{K}-\mathcal{K}_{h}\right)(\underset{\sim}{\boldsymbol{\tau}}, \underset{\sim}{\eta})\right\|_{\hat{\sim}} \underset{\sim}{\hat{\mathbf{Z}}} \leq C h^{\min (2 / r, 2 / s)}\|(\underset{\sim}{\boldsymbol{\tau}}, \underset{\sim}{\eta})\|_{\hat{\sim}} \times \hat{\sim} \underset{\sim}{\hat{\mathbf{z}}},
$$

so that

$$
\left\|\mathcal{K}-\mathcal{K}_{h}\right\|_{\mathcal{L}(\underset{\sim}{\hat{\mathbf{X}}} \times \underset{\sim}{\hat{\mathbf{Z}}}, \underset{\sim}{\hat{\mathbf{X}}} \times \underset{\sim}{\hat{\mathbf{Z}}})} \leq C h^{2 / s},
$$

and this ends the proof.

Therefore, Lemmas 4.2, 5.4 and a classical perturbation argument (cf. 12]) lead to the following result:

Lemma 5.5. Under the assumptions on $\Omega$ of Lemma 5.2 for $h$ small enough, the operator $\left(I+\mathcal{K}_{h}\right)$ is an isomorphism from $\underset{\sim}{\hat{\mathbf{X}}} \times \underset{\sim}{\hat{\mathbf{Z}}}$ into itself. Moreover $\left(I+\mathcal{K}_{h}\right)^{-1}$ maps $\mathbf{X}_{h} \times \mathbf{Z}_{h}$ into itself.

Lemma 5.6. Assume that the assumptions on $\Omega$ of Lemma 5.2 are satisfied. If $(\underset{\sim}{\boldsymbol{\sigma}}, \xi)$ is a nonsingular solution of (37), then there exists a constant $C>0$ such that

$$
\left\|H_{h}\left(\underset{\sim}{\boldsymbol{\sigma}_{h}}, \underset{\sim}{\xi^{*}}\right)\right\|_{\underset{\sim}{\hat{\mathbf{X}}} \times \hat{\mathbf{Z}}} \leq C h^{2 / r}
$$

where ${\underset{\sim}{*}}_{\sigma_{h}}^{*}=\left(\left(\boldsymbol{\sigma}_{h}^{*}, p_{h}^{*}\right) ; \mathbf{u}_{h}^{*}\right)=\left(\Pi_{h}(\boldsymbol{\sigma}, p) ; \mathcal{P}_{h} \mathbf{u}\right)$ and $\xi_{h}{ }^{*}=\left(\xi_{h}^{*}, \theta_{h}^{*}\right)=\left(\Pi_{h}^{0} \xi, \mathcal{P}_{h}^{0} \theta\right)$.

Proof. Since $(\underset{\sim}{\boldsymbol{\sigma}}, \underset{\sim}{\xi})$ is a solution of (37), we may write

$$
\begin{aligned}
& H_{h}\left(\underset{\sim}{\boldsymbol{\sigma}_{h}^{*}, \xi_{h}^{*}}\right)=\left(\begin{array}{c}
\underset{\sim}{\boldsymbol{\sigma}_{\sim}^{*}-\boldsymbol{\sigma}} \\
\underset{\sim}{\xi_{h}{ }^{*}-\underset{\sim}{\xi}}
\end{array}\right)+\left(\begin{array}{cc}
S-S_{h} & 0 \\
0 & L-L_{h}
\end{array}\right)\left(\begin{array}{c}
\mathbf{f}-\frac{1}{\nu}(\boldsymbol{\sigma} \cdot \mathbf{u})-\alpha \theta \\
g-\frac{1}{k}(\mathbf{u} \cdot \xi)
\end{array}\right) \\
& +\left(\begin{array}{cc}
S_{h} & 0 \\
0 & L_{h}
\end{array}\right)\left(\begin{array}{c}
\frac{1}{\nu}\left(\boldsymbol{\sigma}_{h}^{*} \cdot \mathbf{u}_{h}^{*}-\boldsymbol{\sigma} \cdot \mathbf{u}\right)+\alpha\left(\theta_{h}^{*}-\theta\right) \\
\frac{1}{k}\left(\mathbf{u}_{h}^{*} \cdot \xi_{h}^{*}-\mathbf{u} \cdot \xi\right)
\end{array}\right) .
\end{aligned}
$$

From (41), (42), (43), (44), (52) and (53), we have

$$
\begin{gathered}
\left\|\underset{\sim}{\boldsymbol{\sigma}_{h}}-\underset{\sim}{\boldsymbol{\sigma}}\right\|_{\hat{\mathbf{X}} \times \hat{\mathbf{Y}}} \leq C h^{2 / r}, \quad \underset{\sim}{\|} \underset{\sim}{\xi^{*}}-\underset{\sim}{\xi} \|_{\hat{\mathbf{Z}} \times T} \leq C h^{2 / r}, \\
\left\|\left(S-S_{h}\right)\left(\mathbf{f}-\frac{1}{\nu}(\boldsymbol{\sigma} \cdot \mathbf{u})-\alpha \theta\right)\right\|_{\hat{\mathbf{X}} \times \hat{\mathbf{Y}}} \leq C h^{2 / r}, \\
\left\|\left(L-L_{h}\right)\left(g-\frac{1}{k}(\mathbf{u} \cdot \xi)\right)\right\|_{\hat{\mathbf{Z}} \times T} \leq C h^{2 / r} .
\end{gathered}
$$

The term $S_{h}\left(\boldsymbol{\sigma}_{h}^{*} \cdot \mathbf{u}_{h}^{*}-\boldsymbol{\sigma} \cdot \mathbf{u}\right)$ may be written as

$$
S_{h}\left(\boldsymbol{\sigma}_{h}^{*} \cdot \mathbf{u}_{h}^{*}-\boldsymbol{\sigma} \cdot \mathbf{u}\right)=S_{h}\left(\boldsymbol{\sigma}_{h}^{*} \cdot\left(\mathbf{u}_{h}^{*}-\mathbf{u}\right)\right)+S_{h}\left(\left(\boldsymbol{\sigma}_{h}^{*}-\boldsymbol{\sigma}\right) \cdot \mathbf{u}\right) .
$$


Using the fact that $\left\|S_{h} \tilde{\mathbf{f}}\right\|_{\hat{\mathbf{X}} \times \hat{\mathbf{Y}}} \leq C\|\tilde{\mathbf{f}}\|_{0, \Omega}$ for all $\tilde{\mathbf{f}} \in\left(L^{2}(\Omega)\right)^{2}$, (41) and (43), we get

$$
\begin{aligned}
&\left\|S_{h}\left(\boldsymbol{\sigma}_{h}^{*} \cdot\left(\mathbf{u}_{h}^{*}-\mathbf{u}\right)\right)\right\|_{\hat{\mathbf{X}} \times \hat{\mathbf{Y}}} \leq C\left\|\boldsymbol{\sigma}_{h}^{*} \cdot\left(\mathbf{u}_{h}^{*}-\mathbf{u}\right)\right\|_{0, \Omega} \\
& \leq C\left\|\boldsymbol{\sigma}_{h}^{*}\right\|_{0, s, \Omega}\left\|\mathbf{u}_{h}^{*}-\mathbf{u}\right\|_{0, r, \Omega} \quad(s=2 r /(r-2)) \\
& \leq C h^{2 / r}\|(\boldsymbol{\sigma}, p)\|_{1, \Omega}|\mathbf{u}|_{1, \Omega}, \\
&\left\|S_{h}\left(\left(\boldsymbol{\sigma}_{h}^{*}-\boldsymbol{\sigma}\right) \cdot \mathbf{u}\right)\right\|_{\hat{\mathbf{X}} \times \hat{\mathbf{Y}}} \leq C\left\|\left(\boldsymbol{\sigma}_{h}^{*}-\boldsymbol{\sigma}\right) \cdot \mathbf{u}\right\|_{0, \Omega} \\
& \leq C\left\|\boldsymbol{\sigma}_{h}^{*}-\boldsymbol{\sigma}\right\|_{0, r, \Omega}\|\mathbf{u}\|_{0, s, \Omega} \\
& \leq C h^{2 / r}|(\boldsymbol{\sigma}, p)|_{1, \Omega}\|\mathbf{u}\|_{1, \Omega},
\end{aligned}
$$

and

$$
\left\|S_{h}\left(\alpha\left(\theta_{h}^{*}-\theta\right)\right)\right\|_{\hat{\mathbf{X}} \times \hat{\mathbf{Y}}} \leq C(\alpha)\left\|\theta_{h}^{*}-\theta\right\|_{0, \Omega} \leq C(\alpha) h\|\theta\|_{1, \Omega} .
$$

Therefore, these last estimates, with (67), give us

$$
\left\|S_{h}\left(\frac{1}{\nu}\left(\boldsymbol{\sigma}_{h}^{*} \cdot \mathbf{u}_{h}^{*}-\boldsymbol{\sigma} \cdot \mathbf{u}\right)+\alpha\left(\theta_{h}^{*}-\theta\right)\right)\right\|_{\hat{\mathbf{X}} \times \hat{\mathbf{Y}}} \leq C h^{2 / r} .
$$

Finally, a similar procedure leads to

$$
\left\|L_{h}\left(\mathbf{u}_{h}^{*} \cdot \xi_{h}^{*}-\mathbf{u} \cdot \xi\right)\right\|_{\hat{\mathbf{z}} \times T} \leq C h^{2 / r},
$$

and from (64), (65),$(66)$, (68) and the last estimate, we obtain (63).

We are now able to prove the error estimate for nonsingular solutions of (37).

Theorem 5.7. Under the assumptions on $\Omega$ of Lemma 5.2, if $\underset{\sim}{\boldsymbol{\sigma}}, \xi)$ is a nonsingular solution of (37), then for $h$ small enough, problem (48) has at least one solution



$$
\|(\underset{\sim}{\boldsymbol{\sigma}, \xi})-\left(\underset{\sim}{\left.\boldsymbol{\sigma}_{h}, \underset{\sim}{\xi}\right)} \|_{\underset{\sim}{\hat{\mathbf{X}}} \times \hat{\mathbf{z}}} \leq C h^{\frac{4-r}{r}}\right.
$$

where $2<r<4$.

Proof. We define the following map $\mathcal{S}$ from $\underset{\sim}{\mathbf{X}_{h}} \times \underset{\sim}{\mathbf{Z}_{h}}$ into itself

$$
\underset{\sim}{\mathcal{S}\left(\boldsymbol{\tau}_{h}, \underset{h}{\eta}\right)}=\left(\begin{array}{c}
\underset{\sim}{\boldsymbol{\tau}_{h}} \\
\underset{\sim}{\eta_{h}}
\end{array}\right)-\left(I+\mathcal{K}_{h}\right)^{-1} H_{h}\left(\underset{\sim}{\boldsymbol{\tau}_{h}} \underset{\sim}{\underset{\sim}{\eta}}\right)
$$

and prove that it has a fixed point in a neighborhood of $\left(\underset{\sim}{\boldsymbol{\sigma}_{h}}, \xi_{h}{ }^{*}\right)$, where ${\underset{\sim}{\boldsymbol{\sigma}}}_{h}^{*}=$


this end, we start by estimating $\left\|\mathcal{S}\left(\underset{\sim}{\sim} \boldsymbol{\tau}_{h}, \underset{\sim}{\eta_{h}}\right)-\left(\underset{\sim}{\boldsymbol{\sigma}_{h}}, \underset{\sim}{\xi_{h}}{ }^{*}\right)^{t}\right\|_{\underbrace{\hat{\mathbf{X}}}_{\sim} \times \underset{\sim}{\hat{\mathbf{z}}}}$ in terms of 
$\left\|\left(\boldsymbol{\tau}_{\sim}, \underset{\sim}{\eta_{h}}\right)-\left({\underset{\sim}{\boldsymbol{\sigma}_{h}}, \xi_{\sim}^{\xi_{h}}}^{*}\right)\right\|_{\underset{\sim}{\hat{\mathbf{X}} \times \hat{\mathbf{z}}}} \cdot$ We use the notations

$$
(\underset{\sim}{\boldsymbol{\tau}}, \underset{\sim}{\eta})^{t}=\left(\begin{array}{l}
\underset{\sim}{\boldsymbol{\tau}} \\
\underset{\sim}{\eta}
\end{array}\right) \text { and }\left\|(\underset{\sim}{\boldsymbol{\tau}}, \underset{\sim}{\eta})^{t}\right\|_{\hat{\sim}} \times \underset{\sim}{\hat{\mathbf{z}}}=\|(\underset{\sim}{\boldsymbol{\tau}}, \underset{\sim}{\eta})\|_{\hat{\sim}} \times \underset{\sim}{\hat{\mathbf{Z}}} .
$$

We have

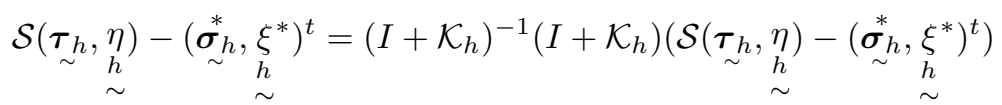

so that

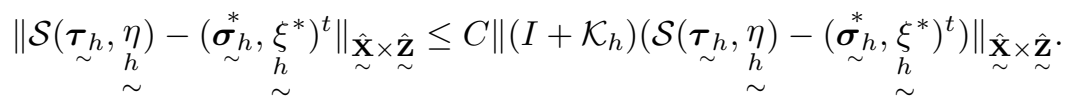

On the other hand,

$$
\begin{aligned}
& \underset{\sim}{\left.\left(I+\mathcal{K}_{h}\right)\left(\mathcal{S}\left(\boldsymbol{\tau}_{h}, \underset{\sim}{\eta}\right)-\underset{\sim}{\sim} \underset{\sim}{*}{\underset{\sim}{h}}^{\xi^{*}}\right)^{t}\right)} \\
& \left.=\left(I+\mathcal{K}_{h}\right)\left(\underset{\sim}{\left(\boldsymbol{\tau}_{h}, \underset{h}{\eta}\right)^{t}}-\underset{\sim}{\sim} \underset{\sim}{\left(\boldsymbol{\sigma}_{h}\right.}, \underset{\sim}{\xi^{*}}\right)^{t}\right)-H_{h}\left(\underset{\sim}{\boldsymbol{\tau}_{h}} \underset{\sim}{\eta}\right)
\end{aligned}
$$

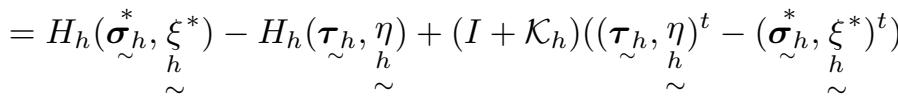

$$
\begin{aligned}
& -H_{h}\left(\underset{\sim}{*} \underset{h}{*}{\underset{\sim}{\xi^{*}}}^{*}\right) \\
& =-\left(\begin{array}{cc}
S_{h} & 0 \\
0 & L_{h}
\end{array}\right)\left(\begin{array}{l}
\frac{1}{\nu}\left(\boldsymbol{\sigma}_{h}^{*}-\boldsymbol{\tau}_{h}\right) \cdot\left(\mathbf{u}_{h}^{*}-\mathbf{v}_{h}\right) \\
\frac{1}{k}\left(\mathbf{u}_{h}^{*}-\mathbf{v}_{h}\right) \cdot\left(\xi_{h}^{*}-\eta_{h}\right)
\end{array}\right)-H_{h}\left(\underset{\sim}{\boldsymbol{\sigma}_{h}} \underset{h}{\xi_{h}^{*}}\right) .
\end{aligned}
$$

Now, using the fact that $\left\|S_{h} \tilde{\mathbf{f}}\right\|_{\hat{\mathbf{X}} \times \hat{\mathbf{Y}}} \leq C\|\tilde{\mathbf{f}}\|_{0, \Omega}$ for all $\tilde{\mathbf{f}} \in\left(L^{2}(\Omega)\right)^{2}$, $\left\|L_{h} g\right\|_{\hat{\mathbf{z}} \times T} \leq C\|g\|_{0, \Omega}$ for all $g \in L^{2}(\Omega)$, and the inverse inequality (cf. [3]) $\left\|\mathbf{v}_{h}\right\|_{0, s, \Omega} \leq C h^{2 / s-2 / r}\left\|\mathbf{v}_{h}\right\|_{0, r, \Omega}$, we have

$$
\begin{aligned}
\left\|S_{h}\left(\left(\boldsymbol{\sigma}_{h}^{*}-\boldsymbol{\tau}_{h}\right) \cdot\left(\mathbf{u}_{h}^{*}-\mathbf{v}_{h}\right)\right)\right\|_{\hat{\mathbf{X}} \times \hat{\mathbf{Y}}} & \leq C\left\|\left(\boldsymbol{\sigma}_{h}^{*}-\boldsymbol{\tau}_{h}\right) \cdot\left(\mathbf{u}_{h}^{*}-\mathbf{v}_{h}\right)\right\|_{0, \Omega} \\
& \leq C\left\|\boldsymbol{\sigma}_{h}^{*}-\boldsymbol{\tau}_{h}\right\|_{0, r, \Omega}\left\|\mathbf{u}_{h}^{*}-\mathbf{v}_{h}\right\|_{0, s, \Omega} \\
& \leq C h^{\frac{2}{s}-\frac{2}{r}}\left\|\boldsymbol{\sigma}_{h}^{*}-\boldsymbol{\tau}_{h}\right\|_{0, r, \Omega}\left\|\mathbf{u}_{h}^{*}-\mathbf{v}_{h}\right\|_{0, r, \Omega} \\
& \leq C h^{\frac{2}{s}-\frac{2}{r}}\left(\left\|\boldsymbol{\sigma}_{h}^{*}-\boldsymbol{\tau}_{h}\right\|_{0, r, \Omega}+\left\|\mathbf{u}_{h}^{*}-\mathbf{v}_{h}\right\|_{0, r, \Omega}\right)^{2}, \\
\left\|L_{h}\left(\left(\mathbf{u}_{h}^{*}-\mathbf{v}_{h}\right) \cdot\left(\xi_{h}^{*}-\eta_{h}\right)\right)\right\|_{\hat{\mathbf{z}} \times T} & \leq C h^{\frac{2}{s}-\frac{2}{r}}\left(\left\|\xi_{h}^{*}-\eta_{h}\right\|_{0, r, \Omega}+\left\|\mathbf{u}_{h}^{*}-\mathbf{v}_{h}\right\|_{0, r, \Omega}\right)^{2},
\end{aligned}
$$

where $s=2 r /(r-2)$. 
Therefore, by (70), (71) and (63), we have

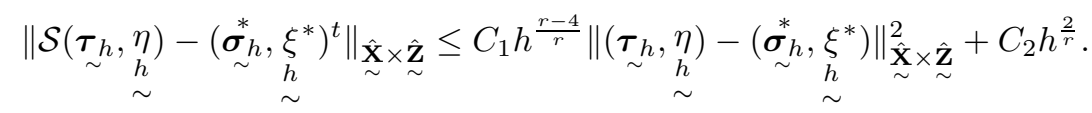

Thus, if

$$
\left\|\left(\underset{\sim}{\boldsymbol{\tau}_{h}}, \underset{\sim}{\eta}\right)-\left(\underset{\sim}{\underset{\sim}{\boldsymbol{\sigma}_{h}}} \underset{\sim}{\xi_{h}^{*}}\right)\right\|_{\underset{\sim}{\mathbf{X}} \times \hat{\mathbf{z}}} \leq \rho(h)
$$

with $\rho(h)$ satisfying

$$
C_{1} h^{\frac{r-4}{r}} \rho^{2}(h)+C_{2} h^{\frac{2}{r}} \leq \rho(h), \quad 2<r<4,
$$

we have

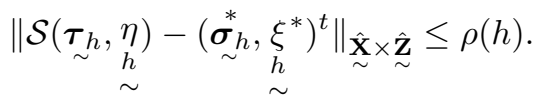

If $h$ is small enough, the greatest root $\rho_{0}(h)$ of the equation

$$
C_{1} h^{\frac{r-4}{r}} \rho^{2}-\rho+C_{2} h^{\frac{2}{r}}=0
$$

satisfies

$$
\rho_{0}(h) \leq C_{1}^{-1} h^{\frac{4-r}{r}},
$$

where $2<r<4$.

Therefore $\mathcal{S}$ has at least a fixed point $\underset{\sim}{\left.\boldsymbol{\sigma}_{h}, \xi_{h}\right)}$ in the ball

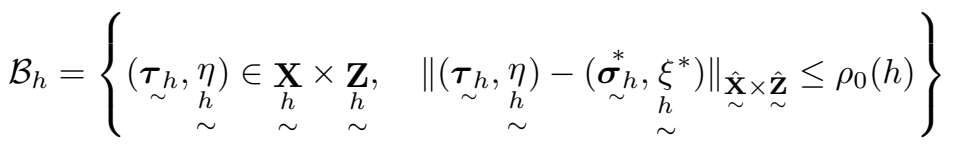

and such a fixed point is a solution of

$$
H_{h}\left(\underset{\sim}{\left.\sigma_{h}, \underset{\sim}{\xi}\right)}=0\right.
$$

Since $\left(\underset{\sim}{\sigma_{h}}, \underset{\sim}{\xi_{h}}\right) \in \mathcal{B}_{h}, \rho_{0}(h) \leq C h^{\frac{4-r}{r}}$ and using (65) , we have the desired result.

\section{REFERENCES}

[1] C. Bernardi, B. Métivet and B. Pernaud-Thomas, Couplage des équations de Navier-Stokes et de la chaleur: Le modèle et son approximation par éléments finis, $\mathrm{M}^{2} \mathrm{AN}, \mathrm{RAIRO}$ Modél. Math. Anal. Numér. 29 (1995), 871-921. MR 96k:76028

[2] F. Brezzi and M. Fortin, Mixed and hybrid finite element methods, Springer-Verlag, New York, 1991. MR 92d:65187

[3] P. Ciarlet, The Finite Element Method for Elliptic Problems, North-Holland, 1978. MR 58:25001

[4] M. Dauge, Elliptic boundary value problems in corner domains. Smoothness and asymptotics of solutions, L.N. in Math., 1341, Springer Verlag, 1988. MR 91a:35078

[5] M. Dauge, Stationary Stokes and Navier-Stokes systems on two- or three-dimensional domains with corners. Part I: Linearized equations, SIAM J. Math. Anal., 20 (1989), 74-97. MR 90b:35191

[6] M. Farhloul, Métdes d'éléments finis mixtes et des volumes finis, Thèse, Université Laval, Québec, 1991.

[7] M. Farhloul, Mixed and nonconforming finite element methods for the Stokes problem, Can. Appl. Math. Quart., 3 (1995) 399-418. |MR 97a:76082

[8] M. Farhloul and M. Fortin, A new mixed finite element for the Stokes and elasticity problems, SIAM J. Numer. Anal., 30 (1993), 971-990. MR 94g:65123 
[9] M. Farhloul and H. Manouzi, Analysis of non-singular solutions of a mixed Navier-Stokes formulation, Comput. Methods Appl. Mech. Engrg., 129 (1996), 115-131. MR 97a:76082

[10] M. Farhloul, S. Nicaise and L. Paquet, A mixed formulation of Boussinesq equations: Analysis of non-singular solutions, Math. Comp. (to appear). CMP 99:10

[11] J.-M. Ghidaglia, Etude d'écoulements de fluides incompressibles: comportement pour les grands temps et applications aux attracteurs, Thèse, Université de Paris-Sud, Centre d'Orsay, France, 1984.

[12] V. Girault and P.-A. Raviart, Finite Element Methods for Navier-Stokes Equations, Springer-Verlag, Berlin, 1986. MR 88b:65129

[13] P. Grisvard, Elliptic problems in nonsmooth domains, Monographs and Studies in Mathematics 21, Pitman, Boston, 1985. MR 86m:35044

[14] L. Paquet, The Boussinesq equations in the presence of thermocapillarity at some part of the boundary, in: G. Lumer, S. Nicaise and B.-W. Schulze eds., Partial Differential Equations, Mathematical Research, 82, Akademie Verlag, Berlin, pp. 266-278, 1994. MR 96a:35146

[15] P.-A. Raviart and J.-M. Thomas, A mixed finite element method for 2-nd order elliptic problems, Lecture Notes in Mathematics, 606, Springer-Verlag, New-York, pp. 292-315, 1977. MR 58:3547

[16] N. Rouche and J. Mawhin, Equations différentielles ordinaires, Tome II: Stabilité et solutions périodiques, Masson, 1973. MR 58:1318b

Université de Moncton, Département de Mathématiques et de Statistique, N.B., E1A 3 E9, Moncton, Canada

E-mail address: farhlom@umoncton.ca

Université de Valenciennes et du Hainaut Cambrésis, LimaV, ISTV, B.P. 311, F-59304

- Valenciennes Cedex, France

E-mail address: snicaise@univ_valenciennes.fr

Université de Valenciennes et du Hainaut Cambrésis, LimaV, ISTV, B.P. 311, F-59304

- Valenciennes Cedex, France

E-mail address: Luc.Paquet@univ_valenciennes.fr 